\title{
On the construction of the Kolmogorov normal form for the Trojan asteroids
}

\author{
Frederic Gabern $^{1,3}$, Àngel Jorba ${ }^{1}$ and Ugo Locatelli ${ }^{2}$ \\ ${ }^{1}$ Departament de Matemàtica Aplicada i Anàlisi, Universitat de Barcelona, Gran Via 585, \\ 08007-Barcelona, Spain \\ 2 Dipartimento di Matematica, Università degli Studi di Roma 'Tor Vergata', \\ Via della Ricerca Scientifica 1, 00133-Roma, Italy \\ E-mail: gabern@maia.ub.es, angel@maia.ub.es and locatell@mat.uniroma2.it
}

Received 18 October 2004, in final form 22 March 2005

Published 13 May 2005

Online at stacks.iop.org/Non/18/1705

Recommended by A Chenciner

\begin{abstract}
In this paper, we focus on the stability of the Trojan asteroids for the planar restricted three-body problem, by extending the usual techniques for the neighbourhood of an elliptic point to derive results in a larger vicinity. Our approach is based on numerical determination of the frequencies of the asteroid and effective computation of the Kolmogorov normal form for the corresponding torus. This procedure has been applied to the first 34 Trojan asteroids of the IAU Asteroid Catalogue, and it has worked successfully for 23 of them.

The construction of this normal form allows computer-assisted proofs of stability. To show this, we have implemented a proof of existence of families of invariant tori close to a given asteroid, for a high order expansion of the Hamiltonian. This proof has been successfully applied to three Trojan asteroids.
\end{abstract}

PACS numbers: 05.10.-a, 05.45.-a, 45.10.-b, 45.20.Jj, 45.50.Pk, 95.10.Ce

\section{Introduction}

The restricted three-body problem (RTBP) models the motion of a particle under the gravitational attraction of two point masses (in our case, Jupiter and the Sun) that revolve in circular orbits around their common centre of mass. It is usual to take a rotating reference frame with the origin at the centre of mass, and such that the Sun and Jupiter are kept fixed on

\footnotetext{
${ }^{3}$ Present address: Control and Dynamical Systems, California Institute of Technology, Mail Stop 107-81, 1200 East California Blvd, Pasadena, CA 91125, USA.
} 


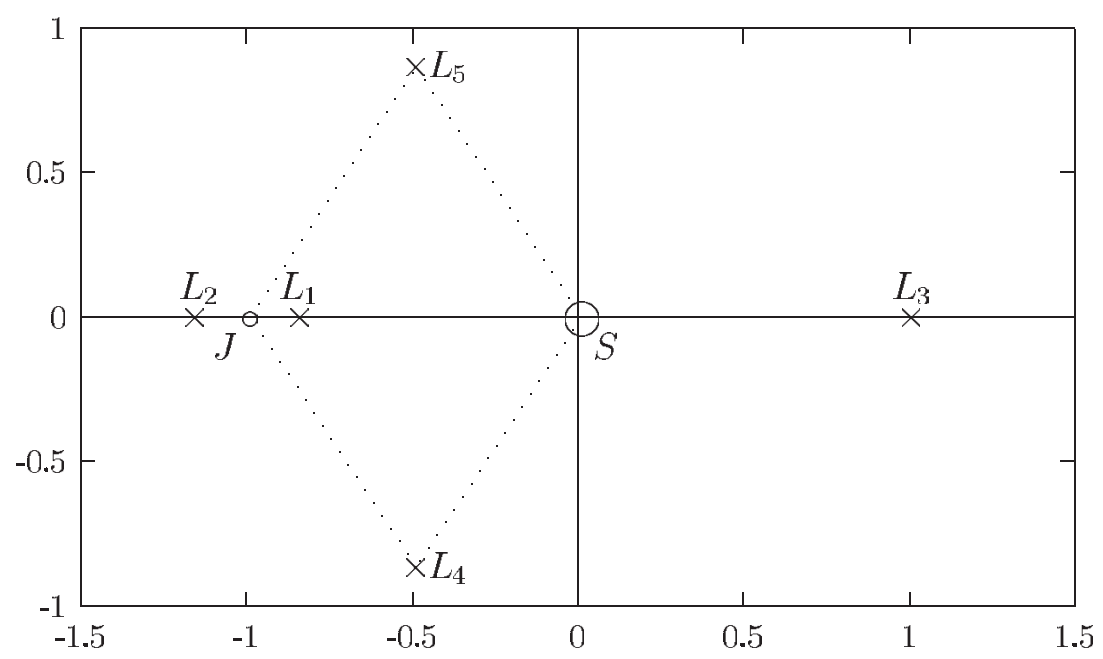

Figure 1. The five equilibrium points of the RTBP.

the $x$ axis, the $(x, y)$ plane is the plane of motion of the primaries and the $z$ axis is orthogonal to the $(x, y)$ plane. These coordinates are usually called synodical. The (adimensional) units are chosen as follows: the unit of distance is the Sun-Jupiter distance, the unit of mass is the total Sun-Jupiter mass and the unit of time is such that the period of Jupiter around the Sun equals $2 \pi$. With this selection of units, it turns out that the gravitational constant is also equal to 1 . Defining momenta as $p_{x}=\dot{x}-y, p_{y}=\dot{y}+x$ and $p_{z}=\dot{z}$, the equations of motion for the particle can be written as an autonomous Hamiltonian system with three degrees of freedom (see [Sze67]):

$$
H_{\mathrm{RTBP}}=\frac{1}{2}\left(p_{x}^{2}+p_{y}^{2}+p_{z}^{2}\right)+y p_{x}-x p_{y}-\frac{1-\mu}{r_{\mathrm{PS}}}-\frac{\mu}{r_{\mathrm{PJ}}},
$$

where $\mu=9.538753600 \times 10^{-4}$ is the mass of Jupiter (in adimensional units), $r_{\mathrm{PS}}^{2}=$ $(x-\mu)^{2}+y^{2}+z^{2}$ is the distance from the particle to the Sun and $r_{\mathrm{PJ}}^{2}=(x-\mu+1)^{2}+y^{2}+z^{2}$ is the distance from the particle to Jupiter. It is also well known that the RTBP has five equilibrium points (see figure 1): the collinear points $L_{1}, L_{2}$ and $L_{3}$ lie on the $x$ axis and the triangular points $L_{4}$ and $L_{5}$ form an equilateral triangle (in the $(x, y)$ plane) with the Sun and Jupiter. The collinear points are of the type centre $\times$ centre $\times$ saddle and the triangular points are linearly stable for $\mu<\mu_{R}=\frac{1}{2}(1-\sqrt{23 / 27}) \approx 0.03852$. The nonlinear stability of the triangular points is a much more difficult problem.

Under very general conditions, the well known KAM theorem [Kol54, Arn63, Mos62] (for a survey, see [AKN88] or [Sev03]) can be applied to a small neighbourhood of the Lagrangian points $L_{4}$ and $L_{5}$ [Leo62, DDB67, Mar71, Mar73, MS86] to ensure the existence of many quasi-periodic motions. Each quasi-periodic trajectory fills densely a compact manifold diffeomorphic to a torus. The union of these invariant tori is a set with positive Lebesgue measure and empty interior. If the motion of the particle is restricted to the $z=p_{z}=0$ plane, then the system only has two degrees of freedom and each torus is a two-dimensional manifold that separates the three-dimensional energy surface, and so it acts as a confiner for the motion - this is the key point in the stability proof for two degrees of freedom Hamiltonian systems. In the spatial case, the energy manifold is five-dimensional and the invariant tori are three-dimensional, so that they cannot act as a barrier for the motion. Hence, it may be possible to have trajectories that wander between these tori and escape from any vicinity 
of an elliptic point. The existence of such trajectories is believed to happen generically in non-integrable Hamiltonian systems. This phenomenon is known as Arnol'd diffusion since it was first conjectured by Arnol'd in [Arn64].

A different approach to the stability of Hamiltonian systems was introduced by Nekhoroshev in [Nek77]. The main idea is to derive an upper bound on the diffusion speed on an open domain of the phase space and to show that the (possible) instability is so slow that it does not show up in practical applications. These techniques have been used to derive bounds for the diffusion near the Lagrangian points (see, for instance, [GDF ${ }^{+} 89$, Sim89, CG91, GS97, SD00, GJ01, GJ05]).

Some of these papers are based on the computation of the so-called Birkhoff normal form [Mos68, MH92]. This is the usual normal form computed when one has a power expansion of a Hamiltonian, vector field or map around an equilibrium point. It is based on near-identity transformations that simplify as much as possible the power expansion up to a given order. This normal form is usually divergent [PM03] and so it is only performed up to a finite order. One of its main properties is that it can be explicitly integrated, so that it provides a careful approximation of the dynamics near the fixed point. The proof of the KAM theorem proposed by Kolmogorov in [Kol54] is based on a different normalizing process (see [dlL01] for a survey). There, it is supposed that the Hamiltonian is written in the so-called action-angle coordinates around an approximately invariant torus. In order to derive a truly invariant torus close to the previous one, one must cancel some terms in the Taylor-Fourier expansion of the Hamiltonian. The Kolmogorov normalizing process is the (infinite) sequence of near-identity transformations that suppresses those terms so that, if it converges, it proves the existence of an invariant torus. The expression of the Hamiltonian after this sequence of transformations is known as the Kolmogorov normal form.

In the exceptional case when the Birkhoff normal form around an elliptic point is convergent, the neighbourhood of this point is completely foliated by invariant tori. Then, given one of these tori, it is possible to change coordinates (about the points $L_{4}$ and $L_{5}$, this can be made by using the so-called Poincaré variables (see section 2.2) plus a translation from the origin to the torus), and the new Hamiltonian is in Kolmogorov normal form around the selected torus (see section 4.1). If the Birkhoff normal form is only known up to a finite order, one cannot immediately provide a Kolmogorov normal form but only an approximation to it, because the selected torus is not invariant. Then, under suitable conditions, it is possible to use the Kolmogorov algorithm [Kol54] to improve this normal form and, in case this process is carried out to the limit, to obtain an invariant torus of the system.

A natural question is the persistence of the stability regions near the Lagrangian points of the Sun-Jupiter RTBP in the real solar system. In 1906, Max Wolf discovered an asteroid (named 588 Achilles) moving near the $L_{4}$ point. Since then, many other asteroids have been found near the triangular points of the Sun-Jupiter system; they are usually called 'Trojans', and their names are chosen from Homer's Iliad.

Even though restricted three-body models are not enough for studying the Trojan problem [GJR04], there have been many attempts to rigorously prove the stability of the motion of some of these asteroids in the RTBP, with rather limited success. The main difficulty is that these proofs are based on the construction of normal forms at the triangular points, and this is only valid in a small neighbourhood of the point. A different approach is developed in [dlLGJV05]: given an approximation to a quasi-periodic motion (i.e. a nearly invariant torus), it can be shown, under general conditions, that there exists a true invariant torus nearby. Here, the role of the perturbing parameter is played by the accuracy of the approximated torus: if the error in the invariance condition is small enough, there is a true invariant torus nearby. Moreover, the result does not require the use of action-angle coordinates and, therefore, the 
hypotheses can be checked, for instance, by means of numerical computations in Cartesian coordinates. The proofs are based on a new iterative scheme that is very suitable for numerics and computer-assisted proofs.

In this work, we extend the classical techniques for the neighbourhood of an elliptic equilibrium point to derive results in a larger vicinity. As a model, we have used the planar RTBP (by planar we mean the restriction of the RTBP to the invariant manifold $z=p_{z}=0$ ). The initial conditions for the Trojan asteroids are obtained by spherical projection on $z=0$ from their initial data in the spatial RTBP. Our approach is based on the following scheme:

(a) expand, up to a high order, the Hamiltonian around the triangular points;

(b) perform a low order Birkhoff normal form;

(c) locate a torus in the normal form with the same frequencies as a given Trojan (the frequencies of the Trojan have been previously obtained by a frequency analysis);

(d) translate the origin to this torus;

(e) complete a Kolmogorov normal form up to high order.

Some of the asteroids are rather far from the triangular points, and it is not possible to obtain a good approximation for their motion by means of the normal form computed in step (b). In these cases, the algorithm constructing the Kolmogorov normal form is typically divergent, because of numerical instabilities in the determination of the translations that are requested at step (e). This is a peculiar phenomenon of the RTBP, as we shall discuss later on. To deal with this problem, we will modify steps (d) and (e) to first construct an intermediate object (i.e. a 'quasi-invariant' torus) for which a low order Kolmogorov normalization can be completed, by avoiding the numerical instabilities. Then, if this intermediate object is carefully chosen so that it is close enough to the targeted torus, we can restart from there the complete construction of the Kolmogorov normal form related to the wanted torus (this procedure resembles a numerical 'continuation' of the normal form from the equilibrium point to the final torus). We stress that this technique only works for tori that are not too far from the equilibrium point.

With the frequency analysis method (GMS02, Las99]), we have computed the basic frequencies of the first 34 asteroids of the IAU Asteroid Catalogue. Four of these asteroids have a chaotic motion, and so we have discarded them. For the remaining 30 asteroids, we have applied the scheme above and succeeded in 23 cases. The seven failures of the method seem to correspond to asteroids that are too far from the triangular points.

Then, the techniques developed in [LG00] have been adapted to compute the Kolmogorov normal form and to derive a computer-assisted proof for the existence of a family of tori very close to a selected asteroid. We remark that the computer-assisted proof has been done for a high order expansion of the RTBP, not for the RTBP itself. The main reason is that taking a high order expansion as the initial Hamiltonian makes the computer-assisted proof much simpler. On the other hand, the differences between a high order expansion and the true RTBP are very small (they are quantified later on), much smaller than the differences between the planar RTBP and the real system.

This paper is organized as follows: in section 2, we describe the preliminary transformations that we make to the RTBP Hamiltonian and how the initial conditions of the Trojan asteroids are obtained from their actual positions and velocities. In section 3 , we use the frequency analysis to make a numerical study of the stability region around the triangular points. In section 4 , the method of constructing invariant tori by means of the Kolmogorov normalization is described. In section 5, the results of applying this method to the Trojan problem are explained in detail. In section 6, we discuss the computer-assisted proof of the stability of some of these asteroids based on the KAM theorem. Finally, in section 7 the conclusions are presented. 


\section{Description of the model}

\subsection{Preliminary transformations on the Hamiltonian}

It is convenient (see [GS97]) to start by performing a preliminary change to heliocentric polar coordinates. We translate the origin from the centre of masses to the Sun, and we introduce polar coordinates by means of the following change of variables:

$$
\begin{aligned}
& x=\rho \cos \theta+\mu, \quad p_{x}=p_{\rho} \cos \theta-\frac{\sin \theta}{\rho} p_{\theta}, \\
& y=\rho \sin \theta, \quad p_{y}=p_{\rho} \sin \theta+\frac{\cos \theta}{\rho} p_{\theta}+\mu .
\end{aligned}
$$

Afterwards, we take local coordinates $\left(X, Y, P_{X}, P_{Y}\right)$ centred in the $L_{4}$ or $L_{5}$ triangular points by performing the following translation:

$$
\begin{aligned}
& \rho=X+1, \quad p_{\rho}=P_{X}, \\
& \theta=Y+\pi \pm \frac{\pi}{3}, \quad p_{\theta}=P_{Y}+1,
\end{aligned}
$$

where the positive sign in the second equation corresponds to $L_{4}$ and the negative sign to $L_{5}$. In these new variables the Hamiltonian takes the form:

$$
\begin{gathered}
H\left(X, Y, P_{X}, P_{Y}\right)=\frac{1}{2}\left[P_{X}^{2}+\frac{\left(P_{Y}+1\right)^{2}}{(X+1)^{2}}\right]-P_{Y}-\mu(X+1) \cos \left(Y+\pi \pm \frac{\pi}{3}\right)-\frac{1-\mu}{X+1} \\
-\frac{\mu}{\sqrt{(X+1)^{2}+1+2(X+1) \cos (Y+\pi \pm \pi / 3)}} .
\end{gathered}
$$

Note that from a solution $\left(X(t), Y(t), P_{X}(t), P_{Y}(t)\right)$ close to $L_{4}$ we can derive another one, $\left(\tilde{X}(t), \tilde{Y}(t), \tilde{P}_{X}(t), \tilde{P}_{Y}(t)\right)$, close to $L_{5}$ (and vice versa) through the symmetry $(\tilde{X}=X$, $\left.\tilde{Y}=-Y, \tilde{P}_{X}=P_{X}, \tilde{P}_{Y}=-P_{Y}\right)$. Therefore, it is enough to study a neighbourhood of the $L_{5}$ point so that we will use the Hamiltonian (3) with the minus sign and, if the considered asteroid is close to $L_{4}$, we will apply this symmetry to work in a neighbourhood of $L_{5}$.

\subsection{Normalization of the quadratic part of the Hamiltonian}

Skipping a constant term, the previous Hamiltonian can be expanded around $L_{5}$ as a real power series of the form

$$
H\left(X, Y, P_{X}, P_{Y}\right)=\sum_{l=2}^{+\infty} f_{l}\left(X, Y, P_{X}, P_{Y}\right),
$$

where $\forall l \geqslant 2, f_{l}\left(X, Y, P_{X}, P_{Y}\right) \in \mathcal{P}_{l}\left(X, Y, P_{X}, P_{Y}\right)$ and $\mathcal{P}_{l}(\cdot)$ is the space of homogeneous polynomials of degree $l$ in the variables appearing as arguments. It is well known that, if $\mu<(1-\sqrt{23 / 27}) / 2$, there is a class of linear canonical transformations $\left(X, Y, P_{X}, P_{Y}\right)=$ $\mathcal{L}\left(x_{1}, x_{2}, y_{1}, y_{2}\right)$ bringing the quadratic part of the Hamiltonian in normal form,

$H^{(\mathrm{I})}\left(x_{1}, x_{2}, y_{1}, y_{2}\right)=\frac{v_{1}}{2}\left(x_{1}^{2}+y_{1}^{2}\right)+\frac{\nu_{2}}{2}\left(x_{2}^{2}+y_{2}^{2}\right)+\sum_{l=3}^{+\infty} f_{l}^{(\mathrm{I})}\left(x_{1}, x_{2}, y_{1}, y_{2}\right)$,

where $f_{l}^{(\mathrm{I})}\left(x_{1}, x_{2}, y_{1}, y_{2}\right) \in \mathcal{P}_{l}\left(x_{1}, x_{2}, y_{1}, y_{2}\right), \forall l \geqslant 3$. One of these canonical transformations can be calculated as described in section 2.1 of [GS97] (where the linear transformation used in $\left[\mathrm{GDF}^{+} 89\right]$ is adapted to the polar coordinates introduced in (2)). We remark that here we have used a canonical transformation $\mathcal{L}$ that is not the same as the one described in [GS97] 
and that the results in this work do not depend on this choice. In the Sun-Jupiter system, we have $\mu=9.538753571 \times 10^{-4}$ and the frequencies take the values

$v_{1}=-8.0463875714716596 \times 10^{-2}, \quad v_{2}=9.9675752553214603 \times 10^{-1}$.

We introduce action-angle variables by means of the canonical transformation $(x, y)=$ $\mathcal{A}(I, \varphi)$ defined as

$$
x_{j}=\sqrt{2 I_{j}} \cos \varphi_{j}, \quad y_{j}=\sqrt{2 I_{j}} \sin \varphi_{j}, \quad \forall j=1, \ldots, n,
$$

where $(I, \varphi)$ are the so-called Poincaré variables and $n$ is the number of degrees of freedom (of course, in the planar RTBP, $n=2$ ). Then, the Hamiltonian can be written as

$$
H^{(\mathrm{II})}(I, \varphi)=v \cdot I+\sum_{l=3}^{+\infty} f_{l}^{(\mathrm{II})}(I, \varphi),
$$

where the functions $f_{l}^{(\mathrm{II})}$ are homogeneous polynomials of degree $l$ in the square root of the actions and trigonometric polynomials of degree $l$ in the angles. Moreover, for any fixed index $m \in\{1, \ldots, n\}$ and for all terms appearing in the expansion of $f_{l}^{(\text {II) }}$ the degree in $\sqrt{I_{m}}$ and the $m$ th component of the Fourier harmonics have the same parity, i.e.

$$
\begin{aligned}
f_{l}^{(\mathrm{II})}(I, \varphi)= & \sum_{i_{1}+\cdots+i_{n}=l} \sum_{j_{1}=0}^{i_{1}} \cdots \sum_{j_{n}=0}^{i_{n}}\left\{c_{i_{1}, \ldots, i_{n}, j_{1}, \ldots, j_{n}}^{(\mathrm{II})}\left(\prod_{m=1}^{n} \sqrt{I_{m}^{i_{m}}}\right) \cos \left[\sum_{m=1}^{n}\left(i_{m}-2 j_{m}\right) \varphi_{m}\right]\right. \\
& \left.+d_{i_{1}, \ldots, i_{n}, j_{1}, \ldots, j_{n}}^{(\mathrm{II})}\left(\prod_{m=1}^{n} \sqrt{I_{m}^{i_{m}}}\right) \sin \left[\sum_{m=1}^{n}\left(i_{m}-2 j_{m}\right) \varphi_{m}\right]\right\},
\end{aligned}
$$

where $c_{i_{1}, \ldots, i_{n}, j_{1}, \ldots, j_{n}}^{(\mathrm{II})}$ and $d_{i_{1}, \ldots, i_{n}, j_{1}, \ldots, j_{n}}^{(\mathrm{III})}$ are real coefficients.

The action-angle coordinates $(I, \varphi)$ and the Hamiltonian (7) are the usual setting for using the tools of the perturbation theory (Birkhoff normal form, KAM and Nekhoroshev theory, etc).

\subsection{Initial conditions of the Trojan asteroids in the planar RTBP}

The orbital elements of the Trojan asteroids are taken from the Bowell Catalogue [Bow] at the Julian date 2452600.5 (22 October 2002). Afterwards, we send their coordinates to the three-dimensional RTBP system and, finally, we project them spherically to the Sun-Jupiter plane to obtain the initial conditions to be used in the computations. We believe that to simulate the Trojan asteroids in the planar RTBP, a spherical projection (which is equivalent to taking zero inclinations) makes more sense than an orthogonal projection with respect to the $z$ axis. In figure 2 , we plot the synodical $(x, y)$ coordinates corresponding to these initial conditions for the RTBP system. It is remarkable how these projected coordinates shadow the famous 'banana' shape of the stability region found in several numerical investigations [MS81].

In figure 3 , the distribution of these initial conditions in the $\left(I_{1}, I_{2}\right)$ plane is shown. We can see that most of them lie in the domain $\max \left\{I_{1}, I_{2}\right\} \leqslant 0.05$, but it is worth noting that some of them reach very high action values $\left(I_{1} \gg 0.1\right)$. This fact makes it very difficult to use perturbative methods around the equilibrium point, $I_{1}=I_{2}=0$, to prove stability for such distant objects.

In figure 4 , we plot a measure of the distance (given by $\|I\|_{2}=\sqrt{I_{1}^{2}+I_{2}^{2}}$ ) of these initial conditions to the triangular points with respect to the two angles $\left(\varphi_{1}, \varphi_{2}\right)$, where the action-angle variables are defined by (6). This figure suggests that for some particular phases (e.g. $\varphi_{1} \sim 1.3$ or 1.7 and $\varphi_{2} \sim 0.3$ or -2.5 ), the stability regions are larger. The asteroids that reach higher values of the actions can go farther from the equilibrium points. In particular, 


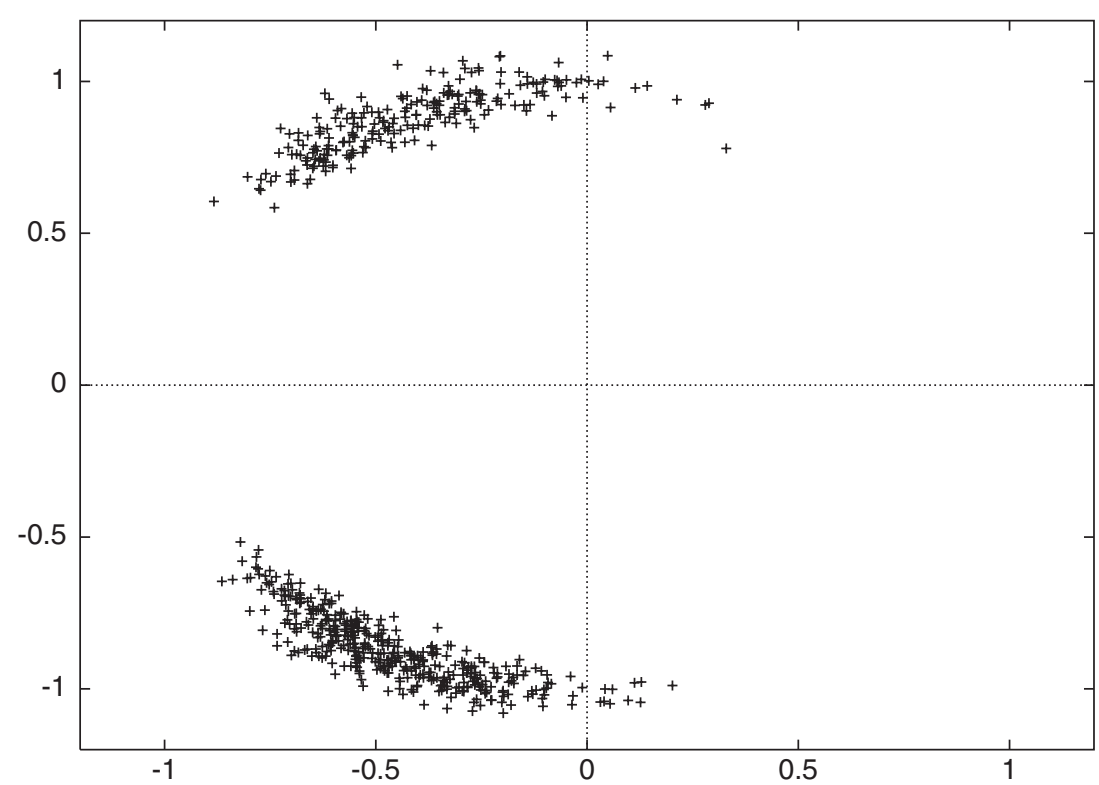

Figure 2. Spherical projection into the $(x, y)$ coordinates of the planar RTBP Sun-Jupiter system of the Trojan orbital elements at the Julian date 2452600.5. Note how these initial conditions shadow the banana shape of the stability region.

they correspond to the bodies that can approach the extremes of the banana region mentioned before. In the next section we confirm these assertions with a numerical simulation of the 'global' dynamics near the triangular region for some concrete phases.

\section{Numerical study of the stability region}

We start by fixing the variables $\varphi_{1}$ and $\varphi_{2}$ to some concrete values (they will be specified later on) and by taking a mesh of points in the positive quadrant of the $\left(I_{1}, I_{2}\right)$ plane:

$$
\begin{array}{ll}
I_{1}=\frac{k_{1} I_{1}^{\max }}{N_{1}} & \text { for } k_{1}=0, \ldots, N_{1}, \\
I_{2}=\frac{k_{2} I_{2}^{\max }}{N_{2}} & \text { for } k_{2}=0, \ldots, N_{2} .
\end{array}
$$

Then, we use these points, $\left(I_{1}, I_{2}, \varphi_{1}, \varphi_{2}\right)$, as initial conditions of a numerical integration in an interval of time $[0, T]$. The integration is performed by the symplectic integrator $\mathcal{S B} \mathcal{A B}_{3}$ (described in [LR01]) with a fixed time step of 0.005 and with $T=2^{15}=32768$ (in adimensional units). Then, by means of a refined Fourier analysis of a sample of the trajectory (see [GMS02] for the actual implemented algorithm and [Las95, Las99] for an introduction to the frequency analysis method), we evaluate the two basic frequencies of the orbits that we call $\omega_{1}=\omega_{1}^{(1)}$ and $\omega_{2}=\omega_{2}^{(1)}$. Afterwards, we repeat the integration in the interval of time $[T, 2 T]$ and we recompute the frequencies. In this case, we call them $\omega_{1}^{(2)}$ and $\omega_{2}^{(2)}$. Finally, we consider the values $\delta_{j}=\left|1-\omega_{j}^{(2)} / \omega_{j}^{(1)}\right|, j=1,2$, as an estimation of the diffusion rate (see [RL01]) related to the orbit starting from the phase space point $\left(I_{1}, I_{2}, \varphi_{1}, \varphi_{2}\right)$. The value of $\delta_{j}$ gives an estimation of the chaoticity of the particular orbit. That is, if the trajectory associated with an initial condition is quasi-periodic, $\delta_{j}$ should be zero. 

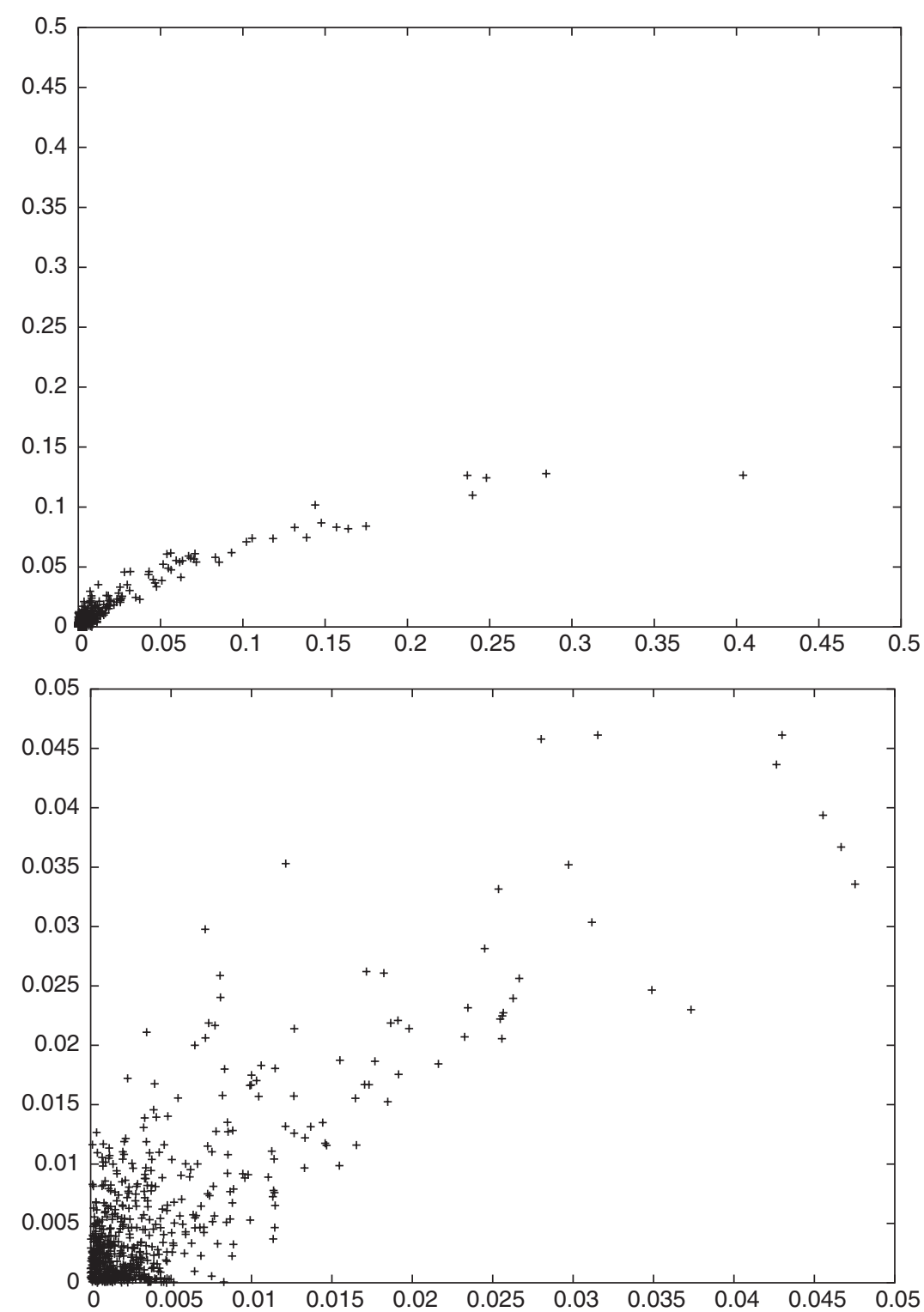

Figure 3. $\left(I_{1}, I_{2}\right)$ spherical projections of the Trojan initial conditions into the Sun-Jupiter plane at the Julian date 2452600.5 . The bottom plot is a zoom of the top plot's left-bottom corner.

In figure 5, we show a contour plot of the function $\sigma_{j}=\log \delta_{j}$ for $j=1$ (we obtain similar pictures for the $\sigma_{2}$ case). The colour code goes from blue (dark grey), corresponding to motion close to quasi-periodic $\left(\delta_{j}<10^{-10}\right)$, to red (light grey), for strongly irregular and escaping motion $\left(\delta_{j}>10^{-2}\right)$. We plot two examples corresponding to the selection of two pairs of phases: $\left(\varphi_{1}, \varphi_{2}\right)=(0,0)$ (left plot) and $\left(\varphi_{1}, \varphi_{2}\right)=(1.78,-2.82)$ (right plot). The stability region (blue part/light grey) shown in the left plot is the generic situation for most of the phases. But, for some particular initial phases, the stability region is much larger. This is the case in the right plot, where we choose a phase very close to the one of the asteroid 2759-Idomeneus (which has a pretty large $\|I\|_{2}$ ). 

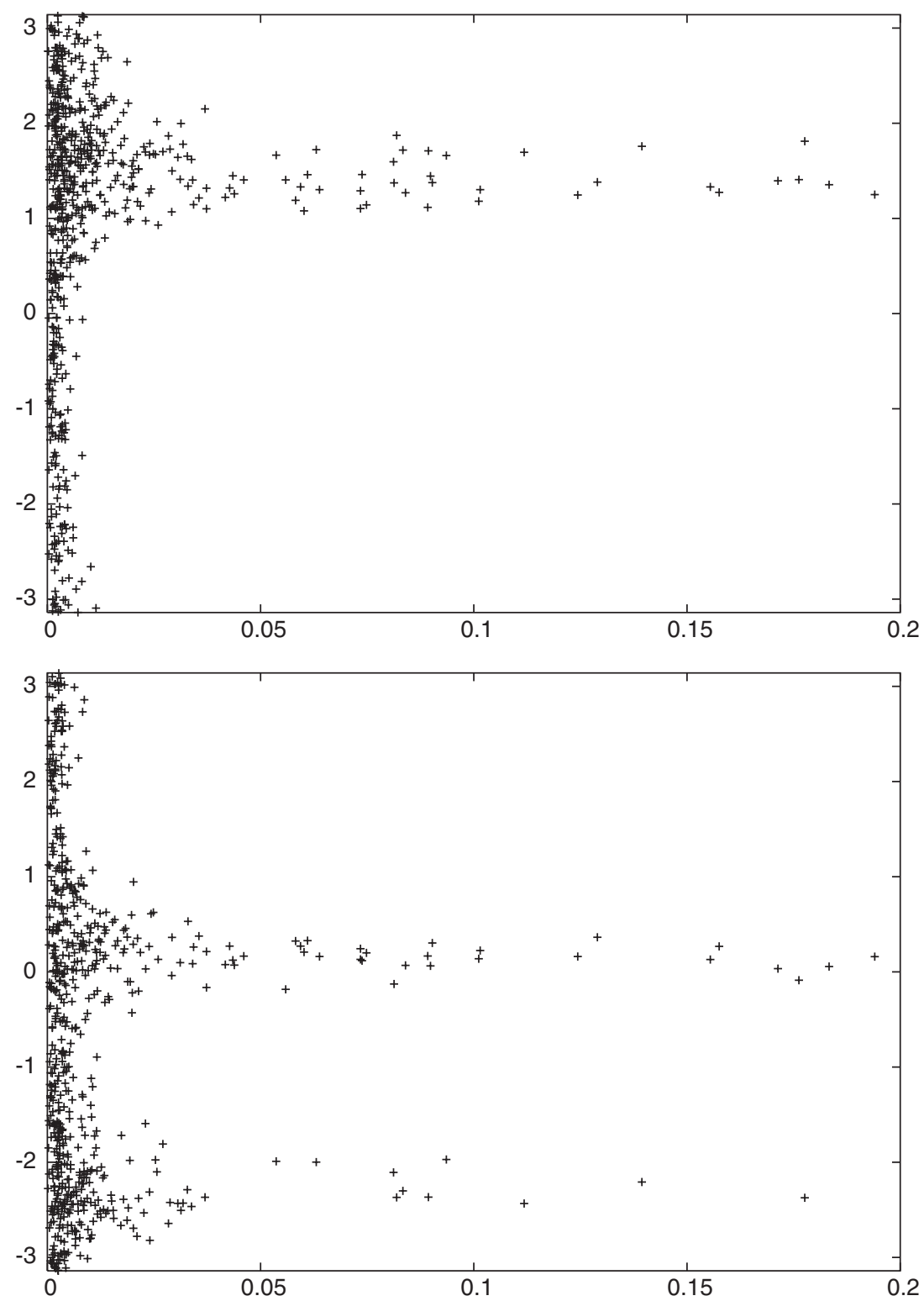

Figure 4. Measure of the distance to the Lagrangian point $\left(\|I\|_{2}=\sqrt{I_{1}^{2}+I_{2}^{2}}\right)$ of the Trojans' projected coordinates. The top plot corresponds to $\left(\|I\|_{2}, \varphi_{1}\right)$ and the bottom one to $\left(\|I\|_{2}, \varphi_{2}\right)$.

Figure 5 highlights two major points when studying the global dynamics near an elliptic equilibrium point, that are the resonances among frequencies and the symmetries of this global picture. The yellow to red (light grey) strips in figure 5 correspond to instabilities generated by resonances between the two basic frequencies, $\omega_{1}$ and $\omega_{2}$ [RGJ05a, RGJ05b]. These resonances, that have been known for a long time [DHR67], are secondary resonances associated with Jupiter's mean motion [RGJ05a] and are due to the linear combinations $p \omega_{1}+\omega_{2}$, with $p=12,13$ and 14 (we checked this by looking at the corresponding plots in the frequency space $\left.\left(\omega_{1}, \omega_{2}\right)\right)$. 

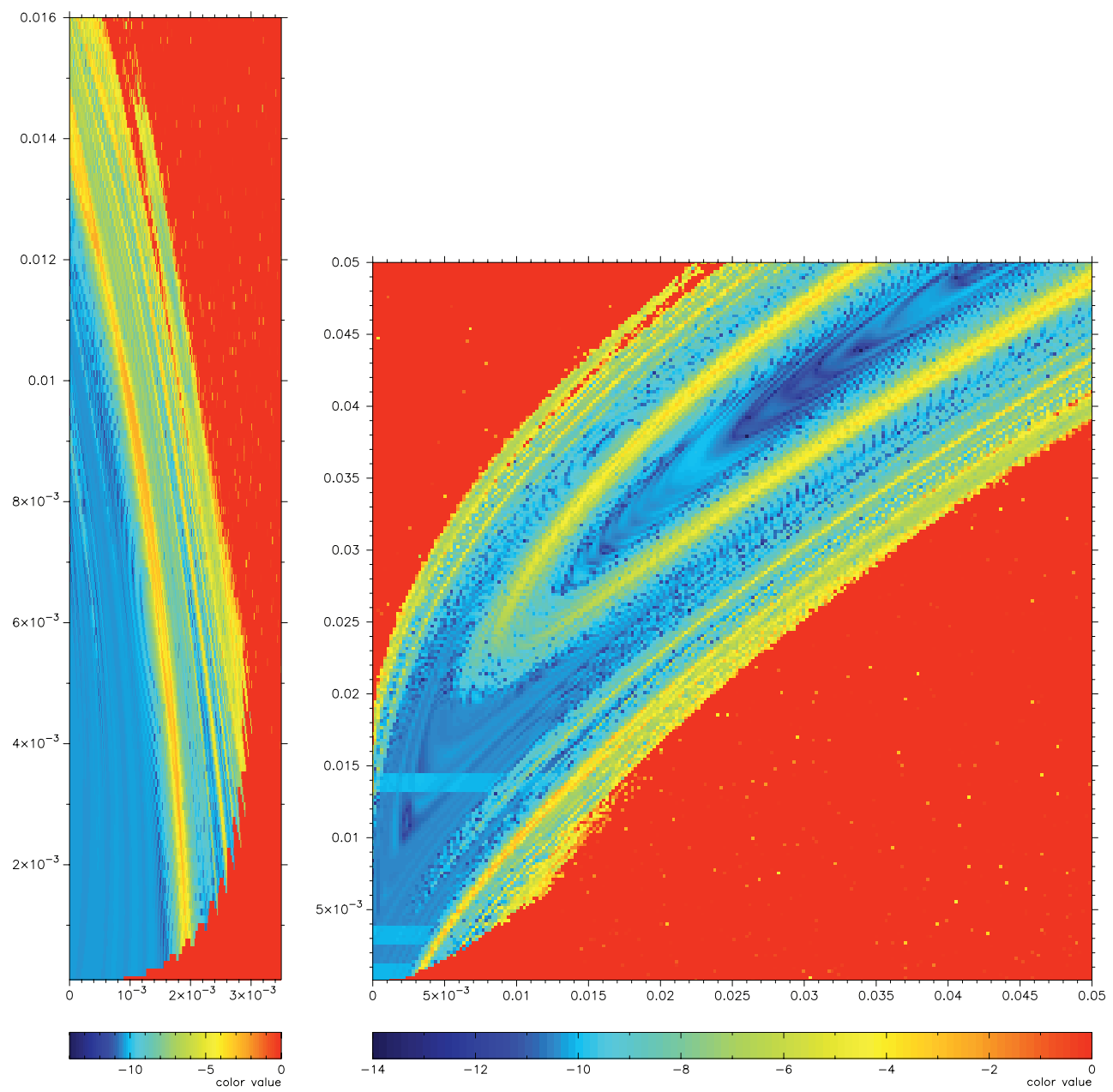

Figure 5. Global stability portrait of the $\left(I_{1}, I_{2}\right)$ plane for two different pairs of phases. The left plot corresponds to the phases $\left(\varphi_{1}, \varphi_{2}\right)=(0,0)$. The phases for the right plot are $\left(\varphi_{1}, \varphi_{2}\right)=(1.78,-2.82)$. See text for more details.

(This figure is in colour only in the electronic version)

On the other hand, figure 5 (right) is clearly symmetric with respect to a (curved) line that crosses the main resonances. As we will show in section 4.2, this symmetry corresponds to the degeneracy of the frequency map. This degeneracy will add some technical difficulties in the construction of the prescribed invariant tori, that can be solved by modifying the usual Kolmogorov normalization algorithm (see section 4.3).

Let us compare the existing analytical results with the stability region given by our numerical investigations. Looking at table 1 of both [GS97] and [SD00] (that contain, to our knowledge, the best existing results of the Trojan stability in the RTBP model), the domain of initial conditions corresponding to orbits that are effectively stable is approximately contained in the rectangle $\left(I_{1}, I_{2}\right) \in[0,0.0005] \times[0,0.0008]$. This fact clearly shows that the analytical results obtained up to now are quite far from giving a complete explanation of the stability of the motion of the Trojans. 


\section{Constructive algorithm for invariant tori close to an elliptic point}

An explicit procedure for constructing invariant tori near an elliptic equilibrium point is described in [LG00], where the considered system is the secular part of the Sun-Jupiter-Saturn system. The algorithm can also be applied to the neighbourhood of the Lagrangian points of the RTBP, but it only converges for initial data in a domain that has more or less the same size as the set of initial conditions for which the method used in [GS97] and in [SD00] works. In this section, we will explain how such a procedure can be modified in order to increase its domain of application. Therefore, in section 4.1 we first adapt the algorithm described in [LG00] to this context. In section 4.2 we provide examples that illustrate why a direct application of that algorithm to the RTBP gives poor results. Finally, in section 4.3 we show the modifications that will allow us to construct invariant tori in a much wider neighbourhood of the equilibrium point.

\subsection{Kolmogorov normalization near an elliptic equilibrium point}

The goal is to introduce a suitable set of coordinates such that (7) is in the Kolmogorov normal form,

$$
H^{(\infty)}(p, q)=\omega \cdot p+\mathcal{O}\left(p^{2}\right),
$$

where the surface $p=0$ is invariant with respect to the flow and the motion over that torus has angular frequencies equal to $\omega \in \mathbb{R}^{n}$. The algorithm consists of a sequence of canonical transformations that we describe in three separate steps.

(i) Birkhoff normalization up to a finite degree. The aim of each step of the Birkhoff normalization is to eliminate the dependence on the angles in the part of the Hamiltonian having a fixed degree in the (square root of the) actions. To be more precise, let us describe the Birkhoff normalization of the third degree. We first determine a generating function $B^{\text {(III) }}$ by solving the equation

$$
v \cdot \frac{\partial B^{(\mathrm{III})}}{\partial \varphi}+f_{3}^{(\mathrm{II})}-\left\langle f_{3}^{(\mathrm{II})}\right\rangle_{\varphi}=0,
$$

where $\langle\cdot\rangle_{\varphi}$ indicates the average over the angles $\varphi$. From the generic form of the functions $f_{l}^{(\mathrm{II})}$ described in (8), it follows that $\left\langle f_{3}^{(\mathrm{II})}\right\rangle_{\varphi}=0$. Using the formalism of the Lie series (for an introduction to these topics, see, e.g., [Grö60] and [Gio95]), the transformed Hamiltonian is given by

$$
H^{(\mathrm{III})}=\exp L_{B^{(\mathrm{III})}} H^{(\mathrm{II})}=\sum_{j=0}^{+\infty} \frac{1}{j !} L_{B^{(\mathrm{III})}}^{j} H^{(\mathrm{II})}=v \cdot I+\sum_{l=4}^{+\infty} f_{l}^{(\mathrm{III})}(I, \varphi),
$$

where we have renamed the new variables of $H^{(\mathrm{III})}$ again $(I, \varphi)$ (this abuse of notation will be repeated hereafter), and one can easily calculate the expression of $f_{l}^{\text {(III) }}$ as a function of $B^{\text {(III) }}$ and $f_{l}^{(\mathrm{II})}$ just collecting the homogeneous polynomials having the same degree in the square root of the actions. Thus, the functions $f_{l}^{(\text {III) }}$ are of the same type as in (8).

It is well known that this sequence of Hamiltonians and canonical transformations produced by the Birkhoff normalization does not converge on any open neighbourhood of the equilibrium point ([PM03]). Note that the Birkhoff normal form of degree 3 is enough to start the following construction of the Kolmogorov normal form and that the radius of convergence of the Hamiltonian shrinks to zero when the degree of the Birkhoff normalization goes to $\infty$. On the other hand, by performing the Birkhoff normalization up to a degree higher than 3 , we can improve the numerical stability of the calculation of the coefficients appearing in the 
expansions generated by the algorithm. Here, we have computed the Birkhoff normalization up to fifth degree, which is good enough for our purposes.

To fix ideas, let us conclude by describing the construction of the Birkhoff normal form up to the fifth degree. The final Hamiltonian is given by

$H^{(\mathrm{V})}=\exp L_{B^{(\mathrm{V})}} \circ \exp L_{B^{(\mathrm{IV})}} \circ \exp L_{B^{(\mathrm{III})}} H^{(\mathrm{II})}=v \cdot I+f_{4}^{(\mathrm{V})}(I)+\sum_{l=6}^{+\infty} f_{l}^{(\mathrm{V})}(I, \varphi)$,

where (a) the functions $f_{l}^{(\mathrm{V})}$ are homogeneous polynomials of degree $l$ in the square root of the actions $I$ of type (8); (b) the generating function $B^{(\mathrm{IV})}$ is defined by the following equation:

$$
v \cdot \frac{\partial B^{(\mathrm{IV})}}{\partial \varphi}+f_{4}^{(\mathrm{III})}-\left\langle f_{4}^{(\mathrm{III})}\right\rangle_{\varphi}=0
$$

(c) the generating function $B^{(\mathrm{V})}$ is obtained by solving an analogous equation where terms of fifth degree in the square root of the actions and with angular average equal to 0 appear; and finally, (d) $f_{4}^{(\mathrm{V})}=\left\langle f_{4}^{(\mathrm{IIII})}\right\rangle_{\varphi}$.

Let us recall that the canonical transformation $\mathcal{B}$ inducing the Birkhoff normalization up to the fifth degree is explicitly given by

$$
\mathcal{B}(I, \varphi)=\exp L_{B^{(\mathrm{V})}} \circ \exp L_{B^{(\mathrm{IV})}} \circ \exp L_{B^{(\mathrm{III})}}(I, \varphi)
$$

because one immediately sees that $H^{(\mathrm{V})}(I, \varphi)=H^{(\mathrm{II})}(\mathcal{B}(I, \varphi))$ using the exchange theorem for Lie series.

(ii) Initial translation of the actions. The canonical transformation $(I, \varphi)=\mathcal{T}(p, q)$ performing a translation of the actions is of the following type:

$$
I_{j}=p_{j}+I_{j}^{*}, \quad \varphi_{j}=q_{j}, \quad \forall j=1, \ldots, n .
$$

Let us recall that we are constructing an invariant torus with a fixed frequency vector $\omega$. Following [LG00], the initial translation can be determined in such a way that, in the integrable approximation, the quasi-periodic motions on the invariant torus $\left(p=0, q \in \mathbb{T}^{n}\right)$ have angular frequencies $\omega$. Therefore, we determine the vector $I^{*}$ with positive components (recall the canonical transformation in (6)) as the nearest to the origin solution of the following equations:

$$
v_{j}+\frac{\partial f_{4}^{(\mathrm{V})}}{\partial I_{j}}(I)+\frac{\partial\left\langle f_{6}^{(\mathrm{V})}\right\rangle_{\varphi}}{\partial I_{j}}(I)=\omega_{j}, \quad \forall j=1, \ldots, n .
$$

We can write the expansion of $H^{(\mathrm{VI})}(p, q)=H^{(\mathrm{V})}(\mathcal{T}(p, q))$ as follows:

$$
H^{(\mathrm{VI})}(p, q)=\omega \cdot p+\sum_{s \geqslant 0} \sum_{l \geqslant 0} f_{l}^{(\mathrm{VI}, s)}(p, q),
$$

where, $\forall l \geqslant 0$ and $s \geqslant 0, f_{l}^{(\mathrm{VI}, s)}$ is a homogeneous polynomial of degree $l$ in the actions and a trigonometric polynomial of degree either $2 s$ or $2 s-1$ in the angles. For short, let us introduce the symbol $\mathcal{P}_{l, 2 s}$, which denotes the set of functions that are homogeneous polynomials of degree $l$ in the actions and trigonometric polynomials of degree at most $2 s$ in the angles. Thus, $f_{l}^{(\mathrm{VI}, s)} \in \mathcal{P}_{l, 2 s}$. Moreover, using the Cauchy inequalities, one easily sees that the size (of any suitable norm) of $f_{l}^{(\mathrm{VI}, s)}$ can be estimated with an upper bound that is essentially proportional to the $s$ th power of the ratio of $\left\|I^{*}\right\|$ over the analytic radius of convergence of $H^{(\mathrm{V})}$, and it is inversely proportional to the $l$ th power of the minimum component of vector $I^{*}$ (see the discussion in [Loc01]).

At this point, we wish to mention that we have some freedom in the crucial choice of the initial translation vector $I^{*}$, as will be discussed in section 4.3 . 
(iii) The standard Kolmogorov normalization algorithm. Let us describe the generic $r$ th step of the Kolmogorov normalization algorithm. We begin with a Hamiltonian of the type

$$
H^{(r-1)}(p, q)=\omega \cdot p+\sum_{s \geqslant 0} \sum_{l \geqslant 0} f_{l}^{(r-1, s)}(p, q)
$$

where $f_{l}^{(r-1, s)} \in \mathcal{P}_{l, 2 s}, \forall l \geqslant 0$ and $s \geqslant 0$. To fix ideas, we can start with $r=2$ defining $H^{(1)}=H^{(\mathrm{VI})}$.

Since we point to a Hamiltonian of type (9), we must remove the main perturbing terms of degree 0 and 1 in the actions. We will proceed in two separate steps. We first remove part of the unwanted terms via a canonical transformation with generating function $\chi_{1}^{(r)}(q)=X^{(r)}(q)+\xi^{(r)} \cdot q\left(\xi^{(r)} \in \mathbb{R}^{n}\right)$. Thus, we solve with respect to $X^{(r)}(q)$ and $\xi^{(r)}$ the equations

$\omega \cdot \frac{\partial X^{(r)}}{\partial q}(q)+\sum_{s=1}^{r} f_{0}^{(r-1, s)}(q)=0, \quad C^{(r)} \xi^{(r)} \cdot p+f_{1}^{(r-1,0)}(p)=0$,

where the $n \times n$ matrix $C^{(r)}$ is defined by the equation $\frac{1}{2} C^{(r)} p \cdot p=f_{2}^{(r-1,0)}(p)$. A unique solution satisfying $\left\langle X^{(r)}\right\rangle_{q}=0$ exists if the frequencies $\omega$ are non-resonant up to order $2 r$, $k \cdot \omega \neq 0, \forall 0<|k| \leqslant 2 r$ with $k \in \mathbb{Z}^{n}$, and if $\operatorname{det} C^{(r)} \neq 0$. We must now give the expressions of the functions $\hat{f}_{l}^{(r, s)}$ appearing in the expansion of the new Hamiltonian:

$$
\hat{H}^{(r)}(p, q)=\omega \cdot p+\sum_{s \geqslant 0} \sum_{l \geqslant 0} \hat{f}_{l}^{(r, s)}(p, q),
$$

where $\hat{H}^{(r)}=\exp L_{\chi_{1}^{(r)}} H^{(r-1)}$. With this aim, we will redefine many times the same quantity without changing the symbol. In our opinion, such a repeated abuse of notation has two advantages: first, this makes it easier to understand the final calculation of $\hat{f}_{l}^{(r, s)}$ instead of using a single very complicated formula; second, the description of the algorithm is more similar to its translation in a programming code. For instance, mimicking the $C$ language, with the notation $a \hookleftarrow b$ we mean that the previously defined quantity $a$ is redefined as $a=a+b$. Therefore, we initially define

$$
\hat{f}_{l}^{(r, s)}=f_{l}^{(r-1, s)}(p, q) \quad \forall l \geqslant 0 \quad \text { and } \quad s \geqslant 0 .
$$

To take into account the Poisson bracket of the generating function with $\omega \cdot p$, we put

$$
\hat{f}_{0}^{(r, 0)} \hookleftarrow \omega \cdot \xi^{(r)}, \quad \hat{f}_{0}^{(r, s)}=0 \quad \forall 1 \leqslant s \leqslant r .
$$

Then, we consider the contribution of the terms generated by the Lie series applied to each function $f_{l}^{(r-1, s)}$ as follows:

$$
\hat{f}_{l-j}^{(r, s+j r)} \hookleftarrow \frac{1}{j !} L_{\chi_{1}^{(r)}}^{j} f_{l}^{(r-1, s)} \quad \forall l \geqslant 1, \quad s \geqslant 0 \quad \text { and } \quad 1 \leqslant j \leqslant l .
$$

Looking at formulae (21)-(23), one can easily check that $\hat{f}_{l}^{(r, s)} \in \mathcal{P}_{l, 2 s}, \forall l \geqslant 0$ and $s \geqslant 0$. We perform now a 'reordering of the terms', by moving the monomials appearing in the expansion of a function $\hat{f}_{l}^{(r, s)}$ to another, in such a way that the so redefined functions $\hat{f}_{l}^{(r, s)}$ are homogeneous polynomials of degree $l$ in the actions and trigonometric polynomials of degree $2 s$ or $2 s-1$ in the angles, $\forall l \geqslant 0$ and $s \geqslant 0$.

In the second part of the $r$ th step of the Kolmogorov normalization algorithm, by using another canonical transformation, we remove the part of the perturbation up to the order of magnitude $r$ that actually depends on the angles, and it is linear in the actions. Thus, we solve with respect to $\chi_{2}^{(r)}(p, q)$ the equation

$$
\omega \cdot \frac{\partial \chi_{2}^{(r)}}{\partial q}(p, q)+\sum_{s=1}^{r} \hat{f}_{1}^{(r, s)}(p, q)=0
$$


where again the solution exists and it is unique if $\left\langle\chi_{2}^{(r)}\right\rangle_{q}=0$ and the frequencies $\omega$ are non-resonant up to order $2 r$. Analogously to what we have done above, we now provide the expressions of the functions $f_{l}^{(r, s)}$ appearing in the expansion of the new Hamiltonian:

$$
H^{(r)}(p, q)=\omega \cdot p+\sum_{s \geqslant 0} \sum_{l \geqslant 0} f_{l}^{(r, s)}(p, q),
$$

where $H^{(r)}=\exp L_{\chi_{2}^{(r)}} \hat{H}^{(r)}$. We initially define

$$
f_{l}^{(r, s)}=\hat{f}_{l}^{(r, s)}(p, q) \quad \forall l \geqslant 0 \quad \text { and } \quad s \geqslant 0 .
$$

In order to take into account the contribution of the terms generated by the Lie series applied to $\omega \cdot p$, we put

$$
f_{1}^{(r, j r)} \hookleftarrow \frac{1}{j !} L_{\chi_{2}^{(r)}}^{j-1}\left(\omega \cdot \frac{\partial \chi_{2}^{(r)}}{\partial q}\right) \quad \forall j \geqslant 1 .
$$

Then, the contribution of the Lie series applied to the rest of the Hamiltonian $\hat{H}^{(r)}$ implies that

$$
f_{l}^{(r, s+j r)} \hookleftarrow \frac{1}{j !} L_{\chi_{2}^{(r)}}^{j} \hat{f}_{l}^{(r, s)} \quad \forall l \geqslant 0, \quad s \geqslant 0 \quad \text { and } \quad j \geqslant 1 .
$$

Finally, we perform a new 'reordering of the terms', so that at the end the functions $f_{l}^{(r, s)} \in \mathcal{P}_{l, 2 s}$ appearing in the expansion (25) of the new Hamiltonian $H^{(r)}$ are again homogeneous polynomials of degree $l$ in the actions and trigonometric polynomials of degree $2 s$ or $2 s-1$ in the angles, $\forall l \geqslant 0$ and $s \geqslant 0$.

Let us recall that the canonical transformation $\mathcal{K}^{(r)}$ inducing the Kolmogorov normalization up to the step $r$ is explicitly given by

$$
\mathcal{K}^{(r)}(p, q)=\exp L_{\chi_{2}^{(r)}} \circ \exp L_{\chi_{1}^{(r)}} \circ \cdots \exp L_{\chi_{2}^{(2)}} \circ \exp L_{\chi_{1}^{(2)}}(p, q) .
$$

This concludes the $r$ th step of the algorithm that can be iterated further.

Let us end the description of the standard Kolmogorov normalization with a final remark. As a main difference with respect to other papers on KAM theory (i.e. [GL97a, GL97b, CGL00, LG00]), here we have not explicitly written the expansions in a small parameter; instead we have clearly prescribed adding up terms corresponding to different orders of magnitude in the small parameter when the 'reordering of the terms' is performed. The main advantage of this slight modification is in saving computer memory when implementing the calculations. Roughly speaking, it is possible to handle the temporarily defined functions in such a way that, for any fixed degree $l$, one can keep in memory the expansion requested by a single function $\in \mathcal{P}_{l, 2 s}$ instead of that requested by a function $\in \mathcal{P}_{l, 2}$, plus one $\in \mathcal{P}_{l, 4}, \ldots$, plus one $\in \mathcal{P}_{l, 2 s}$. This is an important improvement, because the final accuracy of the results depends on the number of Kolmogorov normalization steps that one can explicitly perform on the computer (see the discussions about both the study of the approximation of the orbits and the computer-assisted proofs of existence of KAM tori in [LG00] and [CGL00], respectively).

\subsection{Non-uniqueness of invariant tori for fixed frequency vectors}

As has been mentioned before, a straightforward application of the Kolmogorov normalization algorithm gives disappointing results. Indeed, when the initial translation vector, $I^{*}$, is not very small, the sequence of the canonical transformations is non-convergent. In particular, the first generating function that shows up a sudden increase of the coefficients in the numerical implementation of the method is the translation of the actions, i.e. $\left\{\xi^{(r)} \cdot q\right\}_{r \geqslant 2}$. Here we will discuss some of the reasons for such behaviour. 


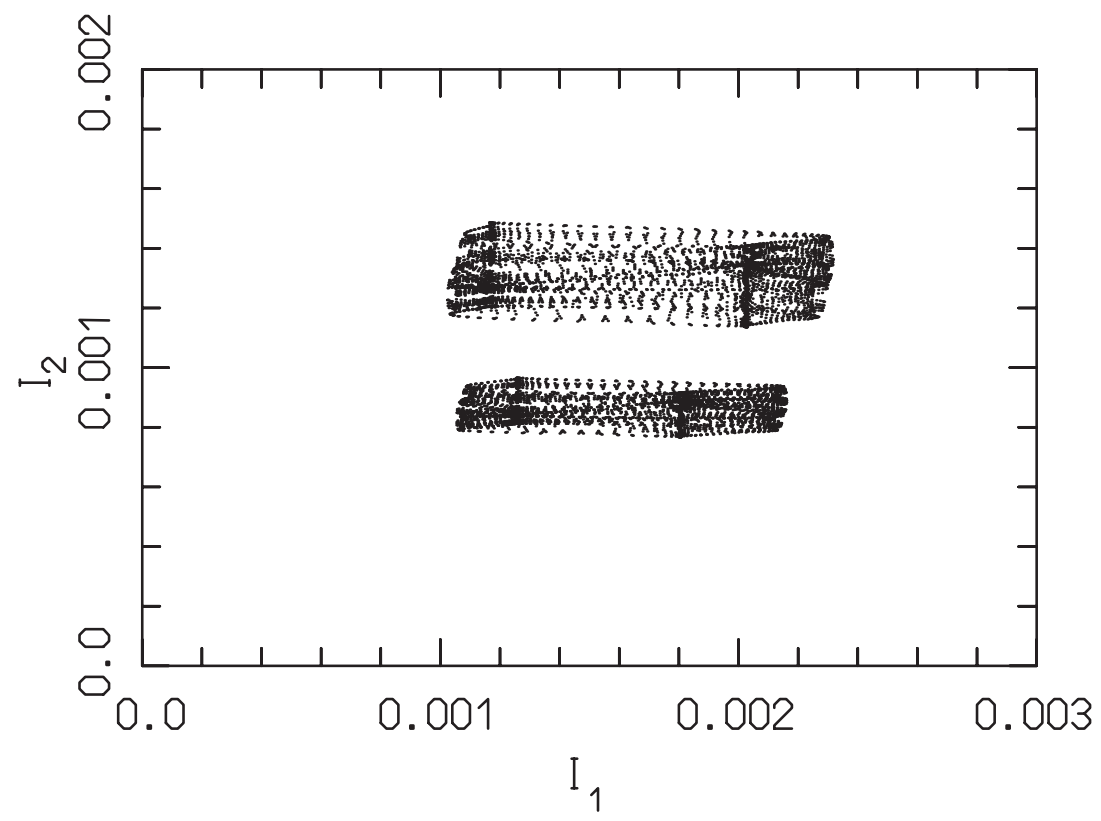

Figure 6. Projection on the plane $\left(I_{1}, I_{2}\right)$ of the shape of two different invariant tori having the same angular frequencies. The lower torus is given by the algorithm described at points (a)-(e) of section 4.3, starting from the frequencies $\left(\omega_{1}, \omega_{2}\right)=$ $(-0.0787481844821318,0.996540006111648)$ corresponding to 624 Hektor (see table 1$)$. The upper torus is obtained by changing the previous algorithm at the point $\left(\mathrm{b}_{1}\right)$ only: in this second case, we used the nearest to the origin solution of (16) as the initial translation vector, $I^{*}$. In both cases, we projected 5000 equally time-spaced points along the motion $(p(t)=0, q(t)=\omega t)$ (where $(p, q)$ are the action-angle coordinates at the end of the normalization procedure) through the canonical transformations $\mathcal{B} \circ \mathcal{T} \circ \mathbf{K}^{(20)} \circ \mathcal{K}^{(20)}$ related to the two different invariant tori (with $\mathcal{B}, \mathcal{T}, \mathbf{K}^{(20)}$ and $\mathcal{K}^{(20)}$ defined as in section 4.3).

First of all, let us recall that the vectors $\xi^{(r)}$ are determined by the second equation in (19), where the matrix $C^{(r)}$ gives approximately (in the limit $r \rightarrow \infty$ ) the local correspondence between actions and frequencies, in a set of coordinates such that the action values locate the invariant tori (see [MG95]).

Figure 6 shows two different tori with the same frequencies: they are related to the construction of the invariant tori corresponding to the orbit of 624 Hektor (as will be discussed in section 4.3). Moreover, we have checked that the determinants of the two matrices $C^{(\infty)}$ related to those two tori have opposite signs. One can prove that the determinant of the matrix $C^{(\infty)}$ is an intrinsic function of the KAM torus. Indeed, canonical transformations for which the manifold $p=0$ is invariant do not change this determinant. Therefore, this is a first indication that there is not a one-to-one mapping between frequencies and invariant tori.

As discussed in [Las99], in the context of quasi-integrable systems, the frequency analysis can be used to describe the diffeomorphism $\Psi: \mathbf{T}^{n} \times \Omega \mapsto \mathbf{T}^{n} \times \mathbf{R}^{n}$ (where $\Omega$ is a suitable open set of frequencies) such that its restriction on a Cantor subset $\tilde{\Omega} \subset \Omega$ locates the invariant tori; i.e. the manifold $\Psi\left(\mathbf{T}^{n}, \omega\right)$ is invariant $\forall \omega \in \tilde{\Omega}$. The existence and regularity of $\Psi$ were proved in [Pös82].

In figure 7 , we numerically study the correspondence between the actions $\left(I_{1}, I_{2}\right)$, before the Birkhoff normalization and the frequencies of the motion $\left(\omega_{1}, \omega_{2}\right)$. More concretely, we investigate the Jacobian, $J$, of $\Phi_{\varphi}(\cdot)=\Psi^{-1}(\varphi, \cdot)$, where the values of the angles $\varphi=\left(\varphi_{1}, \varphi_{2}\right)$ 

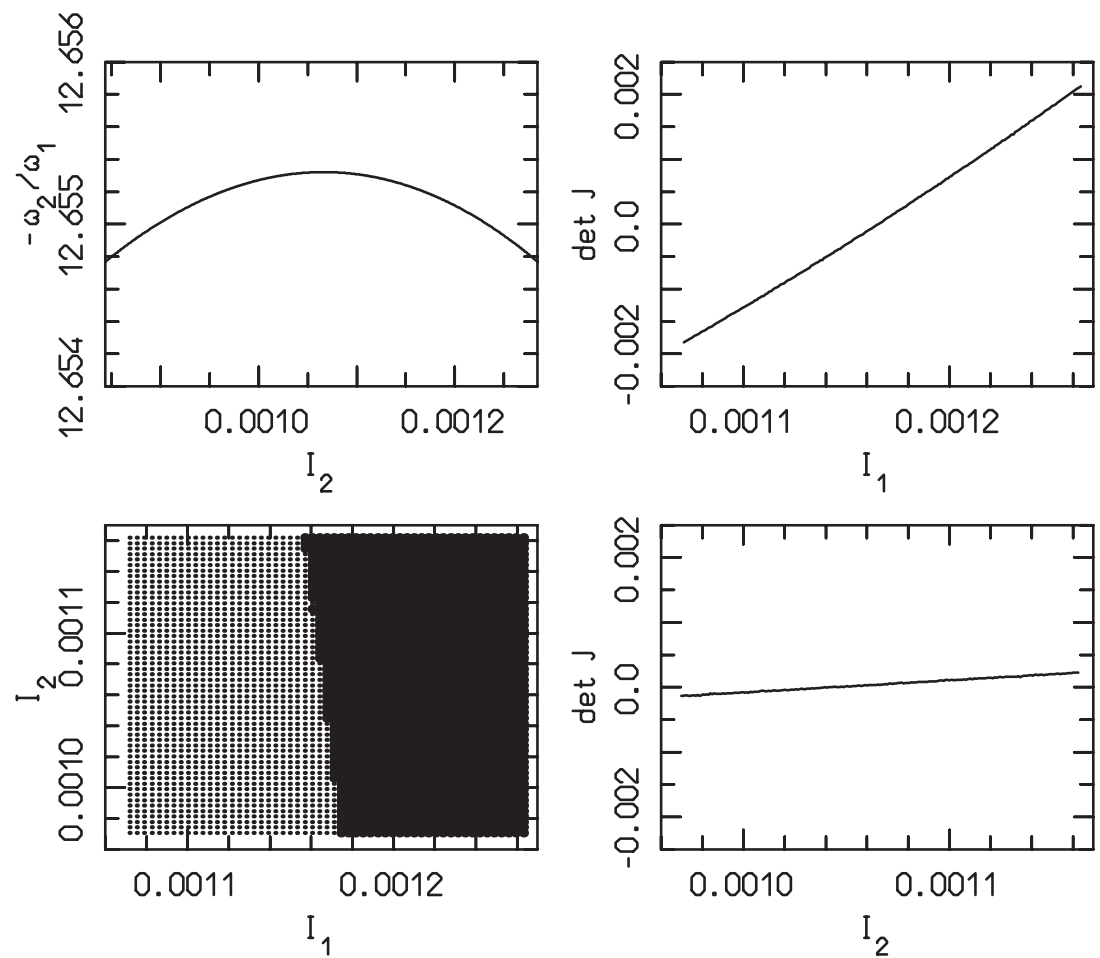

Figure 7. Local study of the Jacobian $J$ of the map associating actions and frequencies. Top-left box: behaviour of the ratio of the frequencies $-\omega_{2} / \omega_{1}$ corresponding to initial conditions on a line connecting the invariant tori in figure 6. Bottom-left box: sign of det $J$ as a function of a grid of initial actions (the initial angles are fixed to the values of the maximum point in the top-left box picture, i.e. $\varphi_{1}=0.205173$ and $\varphi_{2}=0.244$ 193). The region corresponding to non-negative (negative, respectively) values of $\operatorname{det} J$ has been coloured with a darker (lighter) grey. Top-right (bottom-right, respectively) box: section of the function det $J$, after fixing $I_{2}\left(I_{1}\right)$ to its medium value in the same rectangular grid as in the bottom-left box.

are fixed. This figure highlights the existence of a manifold where det $J=0$, for a small set of values of the actions $\left(I_{1}, I_{2}\right)$. Therefore, in this region, the correspondence between frequencies and invariant tori is not invertible.

This is related to the numerical instability of the determination of the translations of the actions, $\left\{\xi^{(r)} \cdot q\right\}_{r \geqslant 2}$. Let us note that in the algorithm described in section 4.1, the determinants of the matrix $C^{(\infty)}$ and the Jacobian $J$ are nearly equal. Indeed, the construction of the Kolmogorov normal form that starts with coordinates $\left(I_{1}, I_{2}, \varphi_{1}, \varphi_{2}\right)$ is given by the composition of:

(i) the Birkhoff normalization $\mathcal{B}$, which is close to the identity in a neighbourhood of the origin of the actions;

(ii) the initial translation of the actions $\mathcal{T}$, that does not change the Jacobian of the frequenciesactions map;

(iii) the standard Kolmogorov normalization algorithm, that uses matrices tending to $C^{(\infty)}$.

Thus, the determinant of $C^{(r)}$ can get very small (possibly zero) when dealing with the normalization algorithm, even for invariant tori located not far from the equilibrium point. We believe that this fact explains the strong numerical instability observed in the determination of the sequence of vectors $\xi^{(r)}$. 

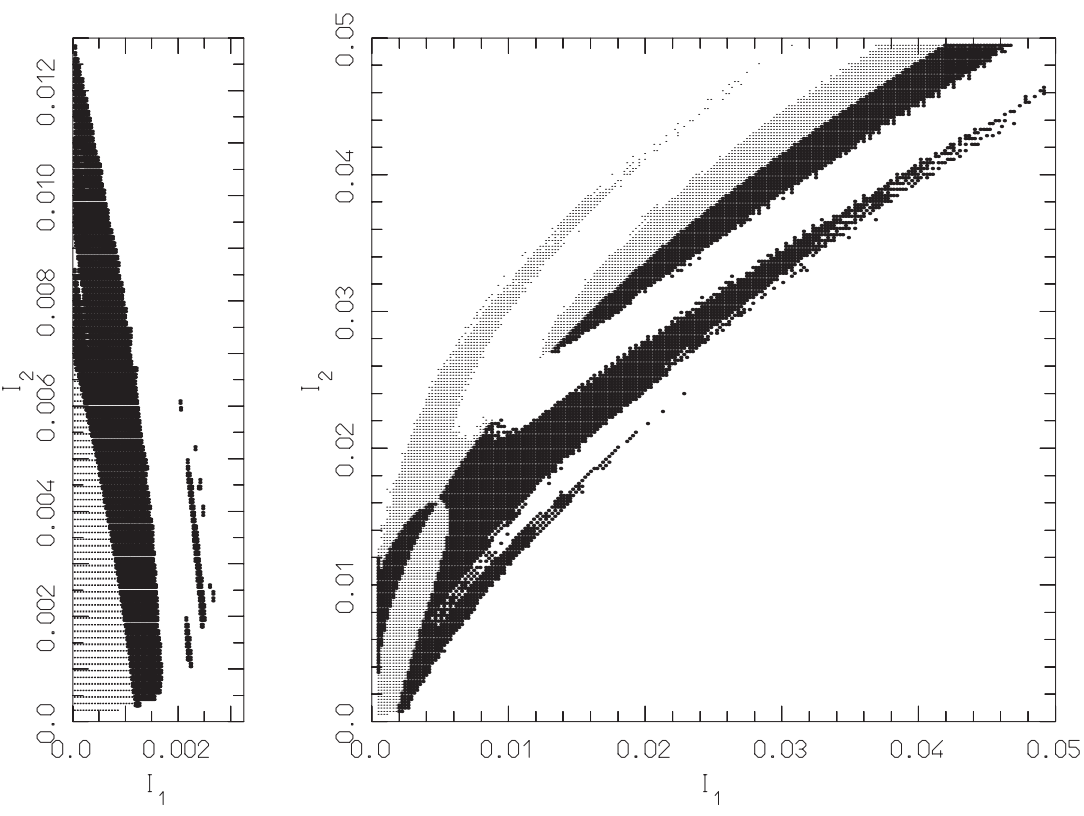

Figure 8. Study of the degeneracy of the map associating actions and frequencies. Both plots report the sign of the determinant of the Jacobian as a function of a grid of initial actions. The left (right, respectively) plot is obtained from the frequency analysis used to produce the left (right) plot of figure 5. The points that do not correspond to quasi-periodic motions in figure 5 (i.e. points such that $\delta_{j}>10^{-9}$ ) are discarded. The region corresponding to non-negative (negative, respectively) values of det $J$ has been coloured with a darker (lighter) grey.

The local study shown in figure 7 describes one of the difficulties in constructing the invariant torus for the particular case of 624 Hektor, that is the degeneracy of the frequency map. In our opinion, this problem would deserve a global analysis, by also using a theoretical approach. Nevertheless, here we just perform a numerical investigation relating the symmetry observed in figure 5 (right) to the degeneracy of this map.

In figure 8, we report the sign of the determinant of the Jacobian as a function of the initial actions for two couples of initial fixed phases (the same ones as in figure 5). The picture on the left, where we fixed the initial phases as $\left(\varphi_{1}, \varphi_{2}\right)=(0,0)$, is the generic one for most values of the angles. Indeed, if we look at the actions considered in the bottom-left box of figure 7 , we see that the location of the manifold where det $J=0$ is approximately the same.

It is clear from figure 8 (right) that the manifold where det $J=0$ approximately coincides with the symmetry line that we observed in figure 5 (right). Indeed, this is the manifold where the frequency map is degenerate. This manifold crosses transversally the resonant region described in section 3 .

\subsection{The modified algorithm constructing the Kolmogorov normal form}

As the prediction of the translation vectors, $\xi^{(r)}$, given by (19) can be affected by large errors, we have split the standard Kolmogorov normalization algorithm into two separate steps. First, we iterate for a fixed number of steps the normalization algorithm by setting to zero the translation vectors $\xi^{(r)}$. This procedure is reminiscent of Arnold's proof of the KAM theorem 
(see [Arn63]). Therefore, under mild theoretical assumptions ${ }^{4}$, such a partial normalization procedure can still converge for $r \rightarrow+\infty$ to a Hamiltonian in Kolmogorov normal form related to a vector of angular frequencies $\omega^{*}$ different from $\omega$. In practice, we perform just a finite number of steps of this partial normalization. Then, using this intermediate Hamiltonian $H \simeq \omega^{*} \cdot p+\mathcal{O}\left(p^{2}\right)$ as the initial one, we restart a complete standard Kolmogorov normalization algorithm now including the translation vectors $\xi^{(r)}$ defined in (19). This splitting of the normalization algorithm into two separate steps becomes advantageous if, after the first step, the frequencies $\omega^{*}$ are sufficiently close to $\omega$ and, thus, the translation vectors $\xi^{(r)}$ are small enough.

Let us remark that the frequencies $\omega^{*}$, related to the intermediate Hamiltonian $H \simeq$ $\omega^{*} \cdot p+\mathcal{O}\left(p^{2}\right)$, depend on the initial translation vector, $I^{*}$, of the canonical transformation (15). Therefore, we try to choose $I^{*}$ in such a way that the frequency vector $\omega^{*}$ is as close as possible to $\omega$. Thus, we use as a first guess of the 'optimal' value of $I^{*}$ the value of the actions of the initial conditions in the coordinates $(I, \varphi)$ after the Birkhoff normalization, because those actions should be 'nearly' constant and then $\left(I=I^{*}, \varphi \in \mathbb{T}^{n}\right)$ should be a 'good enough' initial approximation of the wanted invariant torus. Therefore, we try to improve our choice of $I^{*}$, by approximating numerically the Jacobian matrix $\mathcal{J}_{I^{*}}$ of the function $\omega^{*}\left(I^{*}\right)$ and then solving the linear equation $\mathcal{J}_{I^{*}}\left(\bar{I}-I^{*}\right)=\omega-\omega^{*}$ in the unknown $\bar{I}$. This value, $\bar{I}$, for the initial translation vector is often suitable enough for applying the previous ideas.

We now provide a detailed description of our algorithm. Let us consider as the starting point a system of the type described by the Hamiltonian $H^{(\mathrm{II})}$ in (7). Then, let us carry out the following steps:

(a) Perform the canonical transformation $\mathcal{B}$ that realizes the Birkhoff normalization up to a finite degree, as described at point (i) of section 4.1.

(b) Determine a good initial translation vector $\bar{I}$ by using one step of the following procedure:

$\left(b_{1}\right)$ Let us refer to the initial conditions as $\left(I_{0}, \varphi_{0}\right)$; calculate their values in the new coordinates, say $\left(I_{0}^{*}, \varphi_{0}^{*}\right)=\mathcal{B}\left(I_{0}, \varphi_{0}\right)$. Then, perform the initial translation of the actions, as at point (ii) of section 4.1 , by replacing $I^{*}$ with $I_{0}^{*}$.

$\left(b_{2}\right)$ Let us perform the Kolmogorov normalization algorithm up to a fixed $R^{\prime}$ th step, as at point (iii) of section 4.1, starting from $H^{(1)}=H^{(\mathrm{VI})}$, but putting the translation vectors $\xi^{(r)}=0, \forall r=2, \ldots, R^{\prime}$. Let us define $\omega_{0}^{*}$ in such a way that $\omega_{0}^{*} \cdot p=$ $\omega \cdot p+f_{1}^{(0, r+1)}(p)$, with $f_{1}^{(0, r+1)}$ as obtained at the end of such a procedure. $R^{\prime}$ is a fixed integer parameter that is selected sufficiently large to allow the convergence of the whole algorithm, but also taking into consideration the computational resources available.

( $\left.\mathrm{b}_{3}\right)$ Repeat point $\left(\mathrm{b}_{1}\right)$, by replacing now $I^{*}$ with $I_{0}^{*}+\left(\delta_{1} I_{0}^{*}\right) e_{1}$, where $e_{1}$ is the unit vector in the first axis direction and $\delta_{1} I_{0}^{*}$ is a carefully chosen scalar coefficient such that $\left|\delta_{1} I_{0}^{*}\right| \ll\left\|I_{0}^{*}\right\|$. More concretely, in the numerical applications, we have defined $\delta_{j} I_{0}^{*}=I_{0, j}^{*} / 1000 \forall j=1, \ldots, n$, where $I_{0}^{*}=\left(I_{0,1}^{*}, \ldots, I_{0, n}^{*}\right)$.

$\left(\mathrm{b}_{2 n+1}\right)$ Repeat point $\left(\mathrm{b}_{1}\right)$, by replacing now $I^{*}$ with $I_{0}^{*}+\left(\delta_{n} I_{0}^{*}\right) e_{n}$.

$\left(\mathrm{b}_{2 n+2}\right)$ Repeat point $\left(\mathrm{b}_{2}\right)$, then define $\delta_{n} \omega_{0}^{*}$ in such a way that $\left(\omega_{0}^{*}+\delta_{n} \omega_{0}^{*}\right) \cdot p=$ $\omega \cdot p+f_{1}^{\left(0, R^{\prime}+1\right)}(p)$.

\footnotetext{
${ }^{4}$ In order to prove the convergence of the Kolmogorov normalization algorithm when the translation vectors $\xi^{(r)}$ are neglected, one can adapt the usual technical estimates of the KAM theory. Indeed, in this case it is important to control the displacements of the frequencies due to the contribution of the linear terms (with respect to the actions) in the Hamiltonian. This is certainly possible under the usual hypotheses of the KAM theorem, i.e. the perturbation is small enough, the frequencies belong to a suitable Cantor set of positive Lebesgue measure, etc.
} 
$\left(\mathrm{b}_{2 n+3}\right)$ Let matrix $A$ be such that every $j$ th column is given by $\delta_{j} \omega_{0}^{*} / \delta_{j} I_{0}^{*}, \forall j=1, \ldots, n$; therefore, $A \simeq \mathcal{J}_{I_{0}^{*}}$. Solve the equation $A\left(\bar{I}-I^{*}\right)=\omega-\omega_{0}^{*}$ in the unknown $\bar{I}$.

(c) Perform the translation of the actions as at point (ii) of section 4.1, replacing $I^{*}$ with $\bar{I}$.

(d) Let us perform the Kolmogorov normalization algorithm without translations, as at step $\left(\mathrm{b}_{2}\right)$. In what follows we will denote as $\left\{\mathbf{H}^{(r)}\right\}_{r=1}^{R^{\prime}},\left\{\mathbf{X}_{1}^{(r)}, \mathbf{X}_{2}^{(r)}\right\}_{r=2}^{R^{\prime}}$ and $\left\{\mathbf{K}^{(r)}\right\}_{r=2}^{R^{\prime}}$ the obtained finite sequences of the Hamiltonians, the generating functions and the canonical transformations, respectively, so that $\mathbf{H}^{(1)}=H^{(\mathrm{VI})}, \mathbf{H}^{(r)}=\exp L_{\mathbf{X}_{2}^{(r)}}\left(\exp L_{\mathbf{X}_{1}^{(r)}} \mathbf{H}^{(r-1)}\right)$ and $\mathbf{H}^{(r)}=\mathbf{H}^{(1)} \circ \mathbf{K}^{(r)}, \forall r=2, \ldots, R^{\prime}$. Therefore, $\mathbf{K}^{(r)}$ is explicitly given by a formula analogous to (29), by replacing the symbols $\mathcal{K}$ and $\chi$ with $\mathbf{K}$ and $\mathbf{X}$, respectively.

(e) Let us perform the standard Kolmogorov normalization algorithm, as at point (iii) of section 4.1 (with the translation vectors $\xi^{(r)}$ given by (19)), starting from $H^{(1)}=\mathbf{H}^{\left(R^{\prime}\right)}$.

The knowledge of the normal form (and of the normalizing transformations) allows for an explicit integration of the motion. For instance, if we consider the coordinates $\left(X, Y, P_{X}, P_{Y}\right)$ introduced in section 2.1 and the action-angle variables $(p, q)$ of the normal form, we have that

$$
\begin{gathered}
\left(X(0), Y(0), P_{X}(0), P_{Y}(0)\right) \stackrel{\left(\mathcal{C}^{(\infty)}\right)^{-1}}{\longrightarrow}(p(0)=0, q(0)) \\
\downarrow \\
\left(X(t), Y(t), P_{X}(t), P_{Y}(t)\right) \stackrel{\mathcal{C}^{(\infty)}}{\longleftarrow}(p(t)=p(0), q(t)=q(0)+\omega t)
\end{gathered}
$$

with $\mathcal{C}^{(r)}=\mathcal{L} \circ \mathcal{A} \circ \mathcal{B} \circ \mathcal{T} \circ \mathbf{K}^{\left(R^{\prime}\right)} \circ \mathcal{K}^{(r)}$, where the canonical transformations $\mathcal{L}$ and $\mathcal{A}$ are defined in section 2.2, and $\mathcal{B}, \mathcal{T}, \mathbf{K}^{\left(R^{\prime}\right)}$ and $\mathcal{K}^{(r)}$ are determined at points (a), (c), (d) and (e) of the algorithm described earlier, respectively.

We have checked the implementation by comparing some numerical integrations against the results from the normal form, by means of the scheme (30).

\section{Description of the results}

In figure 9, we have reported the results of such a comparison for 588 Achilles. More precisely, we have derived its initial conditions in the RTBP planar model from a spherical projection of the spatial coordinates. Then we have numerically calculated the corresponding frequencies of motion $\omega=\left(\omega_{1}, \omega_{2}\right)$ by using the frequency analysis method. Afterwards, we have carried out the algorithm constructing the invariant torus corresponding to $\omega$ by performing the Kolmogorov normalization at both points (d) and (e) up to the step $r=R^{\prime}=20$. A software package, specially designed for the algebraic manipulation, allowed us to produce the expansions of the functions defined by the algorithm. The particular choice in the degree of the Kolmogorov normal form (20th order) is due to limitations on both the software and hardware resources available to us, but it is already enough for our purposes. Thus, it has been possible to approximate the motion in a semi-analytic way by using the normal form and the scheme (30), where we have replaced $\mathcal{C}^{(\infty)}$ with $\mathcal{C}^{(20)}$. Finally, we have compared this semi-analytic integration of the equations of motion with a numerical one. The behaviour of the relative difference, $\Delta(X(t), Y(t), \mathbf{P}(t)) /\|(X(t), Y(t), \mathbf{P}(t))\|$ (where, for short, $\mathbf{P}$ means $\left.P_{X}, P_{Y}\right)$, is plotted in the left column of figure 9 , on the time interval $\left[-10^{5}, 10^{5}\right]$, whose width corresponds to about $0.4 \mathrm{My}$. The agreement between the results of the two methods is excellent. Indeed, in terms of the synodical coordinates $(x, y)$, the absolute difference between the values of each coordinate as calculated by the two different methods stays within $5.3 \times 10^{-7}$ (which is equivalent to an error of about $10^{-7} \mathrm{AU}$, comparable with the current uncertainty of the observations). In the top-right box of figure 9, we have projected the invariant torus on the synodical coordinates $(x, y)$. We can appreciate that the invariant torus extends also quite far 

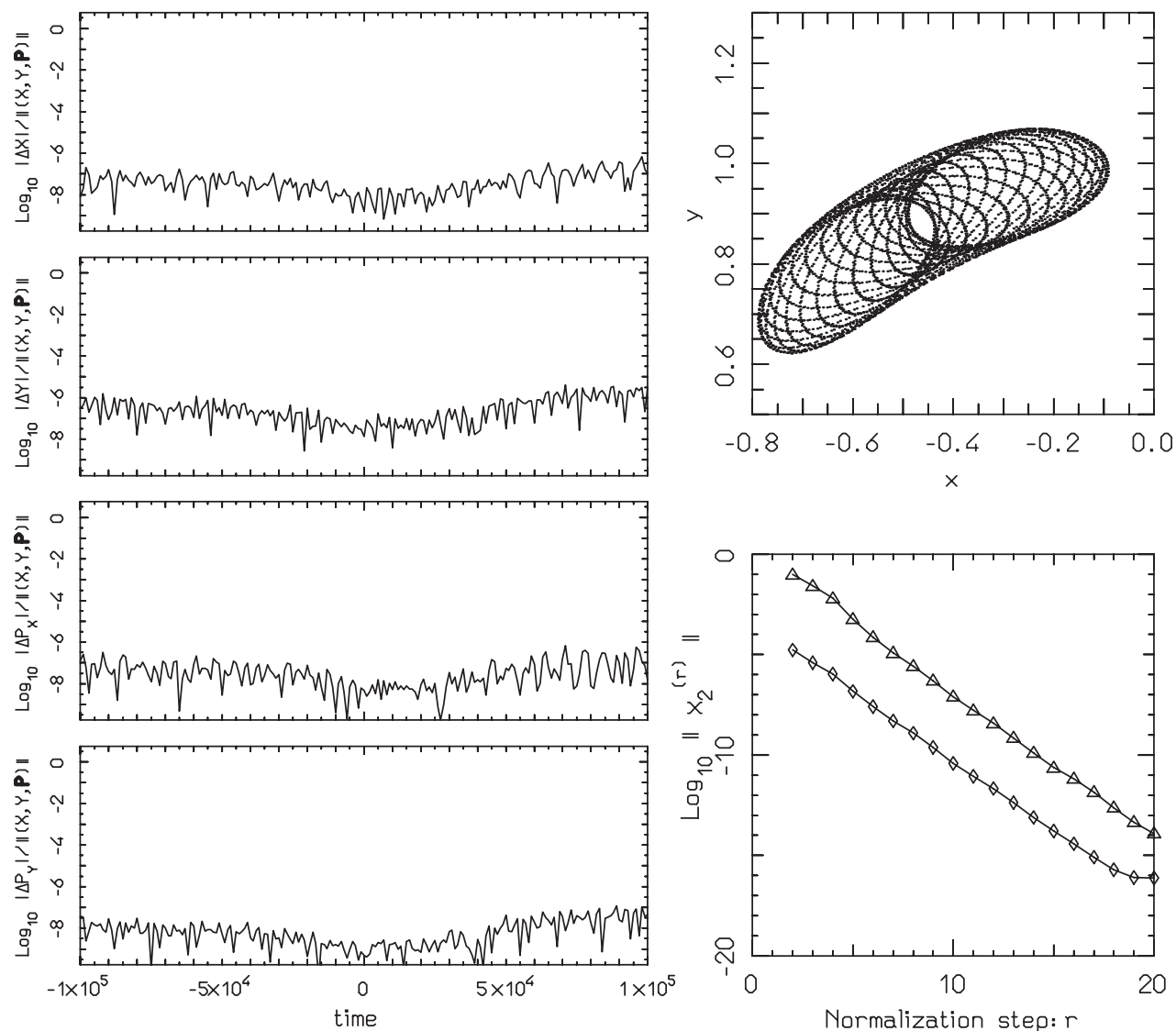

Figure 9. Test on the reliability of the construction of the Kolmogorov normal form on the planar RTBP for the asteroid 588 Achilles. On the left: time dependence of the relative differences between a numerical integration and the semi-analytic one, based on an approximation of the scheme (30) (see the text for more details). Top-right box: projection of the invariant torus in the $(x, y)$ coordinates. Bottom-right box: behaviour of the norm of the generating functions as a function of the normalization step; more precisely, the symbols $\Delta$ ( $\diamond$, respectively) refer to the norm of the generating functions $\mathbf{X}_{2}\left(\chi_{2}\right)$ defined during the Kolmogorov normalization without (with) translations, as described at point (d) (point (e)) of section 4.3 .

away from the equilibrium point (compare with figure 1) and the area filled by its projection is not negligible at all with respect to the region described by the initial conditions of the asteroids (see figure 2). Moreover, in the bottom-right box of figure 9, we reported the norm (given by formula (31)) of the generating functions. We can appreciate a sharp geometrical decreasing of the norms as a function of the normalization step for the generating functions defined by Kolmogorov normalizations with and without the translations.

We have applied this procedure for the first 34 Trojan asteroids of the catalogue. The initial conditions in the RTBP, with spherical projection in the plane $z=0$, give rise to orbits which either have close encounters with Jupiter or are strongly chaotic in four cases (1172 Aneas, 1583 Antilochus, 1873 Agenor and 2223 Sarpedon). Concerning the remaining 30 asteroids, the corresponding motions look quasi-periodic according to the frequency analysis method. Hence, it has been possible to apply the algorithm to constructing the corresponding invariant tori. The algorithm does not work properly for seven other 

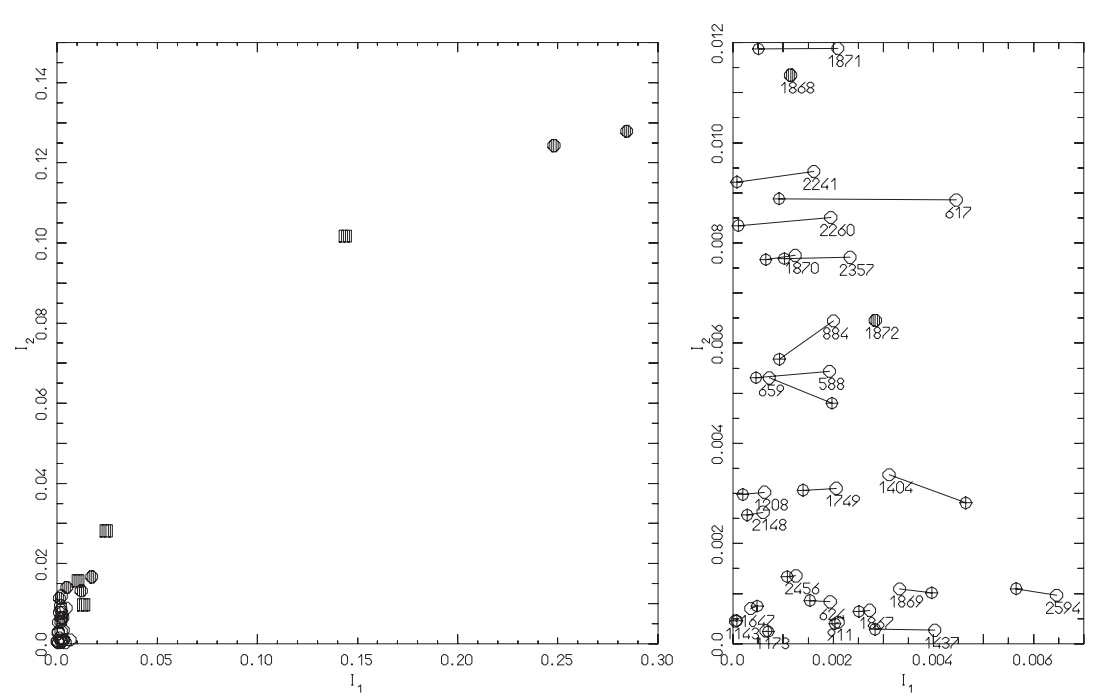

Figure 10. Summary of the results of the algorithm constructing invariant tori. In correspondence with the values of the actions $\left(I_{1}, I_{2}\right)$ related to the initial conditions (expressed in the coordinates $(I, \varphi)$ previous to the Birkhoff normalization), we have used the symbol $\mathbf{w}$ when the orbit is chaotic, - if the algorithm does not converge and $\bigcirc$ if it does. The figure on the right is an enlargement of the one on the left. In addition, in the right figure, below every symbol, we show the catalogue number of the corresponding asteroid. Moreover, for each case where the algorithm converges, the corresponding initial translation vector $\left(\bar{I}_{1}, \bar{I}_{2}\right)$ has been indicated with the symbol $\oplus$.

asteroids: 1868 Thersites, 1872 Helenos, 2146 Stentor, 2207 Antenor, 2363 Cebriones, 2674 Pandarus and 2759 Idomeneus. For all the remaining cases, the sequences of the norms of the generating functions $\mathbf{X}_{2}^{(r)}$ and $\chi_{2}^{(r)}$ (defined in points (d) and (e) of section 4.3, respectively) show a very regular geometrically decreasing behaviour similar to that shown in the bottom-right box of figure 9 . Therefore, the algorithm is clearly convergent for all these 23 asteroids.

The results are visualized in figure 10 , by referring to the same action variables $\left(I_{1}, I_{2}\right)$ used in section 3 to numerically study the stability region close to the equilibrium point. By comparing figure 10 (right) with figure 5 (left), we immediately realize that our algorithm seems to be convergent every time that the initial values of the actions are located in the 'usual' stability region. Indeed, the algorithm does not work when the initial actions are simply too big. Moreover, the convergence of our algorithm can be prevented also for the bodies that are close to a resonance, while their initial conditions are not extremely far from the equilibrium point. For instance, the frequencies of the asteroids 1868 Thersites and 1872 Helenos are quite close to the resonant combinations $13 \omega_{1}+\omega_{2}=0$ and $14 \omega_{1}+\omega_{2}=0$, respectively. Furthermore, by a direct numerical integration, we checked that they can reach much larger distances from the equilibrium point with respect to the initial ones. This can explain why the crucial determination of the 'good initial translation vector' $\bar{I}$ (as in section 4.3) looks very uncertain for those asteroids.

The main results obtained are summarized in table 1 . In the fourth and fifth columns of this table, we have reported the decimal logarithm of the ratios $\left\|\mathbf{X}_{2}^{(20)}\right\| /\left\|\mathbf{X}_{2}^{(2)}\right\|$ and $\left\|\chi_{2}^{(20)}\right\| /\left\|\chi_{2}^{(2)}\right\|$, respectively. Of course, the smaller these ratios are, the faster the convergence of the algorithm is. The initial translation vectors $\bar{I}$ (determined according to the point (b) of section 4.3), are reported in the third column. Let us recall that the size of the perturbation is 


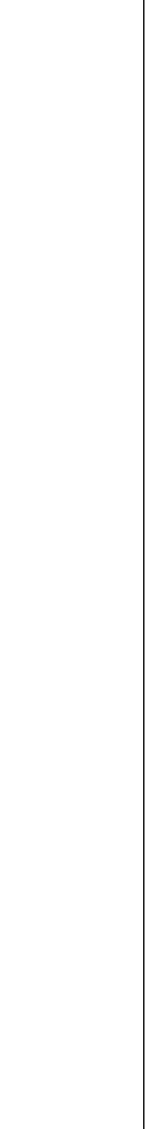




\begin{tabular}{|c|c|c|c|c|c|}
\hline 1647 & $\left(\begin{array}{c}-0.080027652156843390, \\
0.99669299882105700\end{array}\right)$ & $\left(\begin{array}{l}4.8053543816242646 \times 10^{-4}, \\
7.5059131290292904 \times 10^{-4}\end{array}\right)$ & -18.0 & -13.4 & $\left(\begin{array}{l}0.2,2.2, \\
0.1,0.03\end{array}\right) \times 10^{-5}$ \\
\hline 1749 & $\left(\begin{array}{c}-0.079233437316304542, \\
0.99659266795195256\end{array}\right)$ & $\left(\begin{array}{l}1.3960082696097675 \times 10^{-3}, \\
3.0645766576859793 \times 10^{-3}\end{array}\right)$ & -11.8 & -11.4 & $\left(\begin{array}{cc}0.3, & 3.5 \\
0.3, & 0.07\end{array}\right) \times 10^{-5}$ \\
\hline 1867 & $\left(\begin{array}{c}-0.077400216491750912, \\
0.99640034798208466\end{array}\right)$ & $\left(\begin{array}{l}2.5125755624158183 \times 10^{-3}, \\
6.4564726053964829 \times 10^{-4}\end{array}\right)$ & -9.1 & -7.3 & $\left(\begin{array}{l}0.05,1.1, \\
0.09,0.005\end{array}\right) \times 10^{-4}$ \\
\hline 1869 & $\left(\begin{array}{c}-0.075273368358587736, \\
0.99622553356358334\end{array}\right)$ & $\left(\begin{array}{l}3.9658962836038826 \times 10^{-3}, \\
1.0138420157461863 \times 10^{-3}\end{array}\right)$ & -6.9 & -7.0 & $\left(\begin{array}{l}0.07,3.1 \\
0.08,0.002\end{array}\right) \times 10^{-4}$ \\
\hline 1870 & $\left(\begin{array}{c}-0.080795233402109923, \\
0.99675584255254235\end{array}\right)$ & $\left(\begin{array}{l}6.5354209594490167 \times 10^{-4}, \\
7.6674696509944230 \times 10^{-3}\end{array}\right)$ & -9.1 & -8.6 & $\left(\begin{array}{l}1.0,5.4 \\
0.9,0.3\end{array}\right) \times 10^{-6}$ \\
\hline 1871 & $\left(\begin{array}{c}-0.081514040787185243, \\
0.99682673629125285\end{array}\right)$ & $\left(\begin{array}{l}5.0828198584491674 \times 10^{-4}, \\
1.1874638620374238 \times 10^{-2}\end{array}\right)$ & -5.8 & -5.5 & $\left(\begin{array}{l}0.3,1.8 \\
0.7,0.05\end{array}\right) \times 10^{-3}$ \\
\hline 2148 & $\left(\begin{array}{c}-0.080533074925310380, \\
0.99674515743171077\end{array}\right)$ & $\left(\begin{array}{l}2.7971788895957324 \times 10^{-4}, \\
2.5682028661620252 \times 10^{-3}\end{array}\right)$ & -16.4 & -12.6 & $\left(\begin{array}{l}0.9,7.0 \\
1.3,0.2\end{array}\right) \times 10^{-6}$ \\
\hline 2241 & $\left(\begin{array}{c}-0.081707303284590957, \\
0.99685130480612028\end{array}\right)$ & $\left(\begin{array}{l}7.3644442596587732 \times 10^{-5}, \\
9.2156409035582809 \times 10^{-3}\end{array}\right)$ & -10.1 & -7.8 & $\left(\begin{array}{l}1.0,8.0, \\
2.1,0.2\end{array}\right) \times 10^{-7}$ \\
\hline 2260 & $\left(\begin{array}{c}-0.081556086303685743, \\
0.99683754696145255\end{array}\right)$ & $\left(\begin{array}{l}1.0272079815504267 \times 10^{-4}, \\
8.3465843575113198 \times 10^{-3}\end{array}\right)$ & -11.3 & -9.1 & $\left(\begin{array}{l}0.2,1.6, \\
0.3,0.03\end{array}\right) \times 10^{-6}$ \\
\hline 2357 & $\left(\begin{array}{c}-0.080330839991193073, \\
0.99670820475542277\end{array}\right)$ & $\left(\begin{array}{l}1.0216317484861338 \times 10^{-3}, \\
7.6859257344373544 \times 10^{-3}\end{array}\right)$ & -10.0 & -9.1 & $\left(\begin{array}{l}0.6,6.6 \\
1.0,0.3\end{array}\right) \times 10^{-6}$ \\
\hline 2456 & $\left(\begin{array}{c}-0.079390776282647166, \\
0.99661226620203403\end{array}\right)$ & $\left(\begin{array}{l}1.0796571731783104 \times 10^{-3}, \\
1.3388711955805834 \times 10^{-3}\end{array}\right)$ & -13.7 & -11.7 & $\left(\begin{array}{l}0.07,1.5, \\
0.07,0.02\end{array}\right) \times 10^{-4}$ \\
\hline 2594 & $\left(\begin{array}{c}-0.072399117763879486, \\
0.99605155197123274\end{array}\right)$ & $\left(\begin{array}{l}5.6531551656997595 \times 10^{-3}, \\
1.0973030849600765 \times 10^{-3}\end{array}\right)$ & -5.4 & -4.9 & $\left(\begin{array}{l}0.07,1.7 \\
0.1,0.03\end{array}\right) \times 10^{-2}$ \\
\hline
\end{tabular}



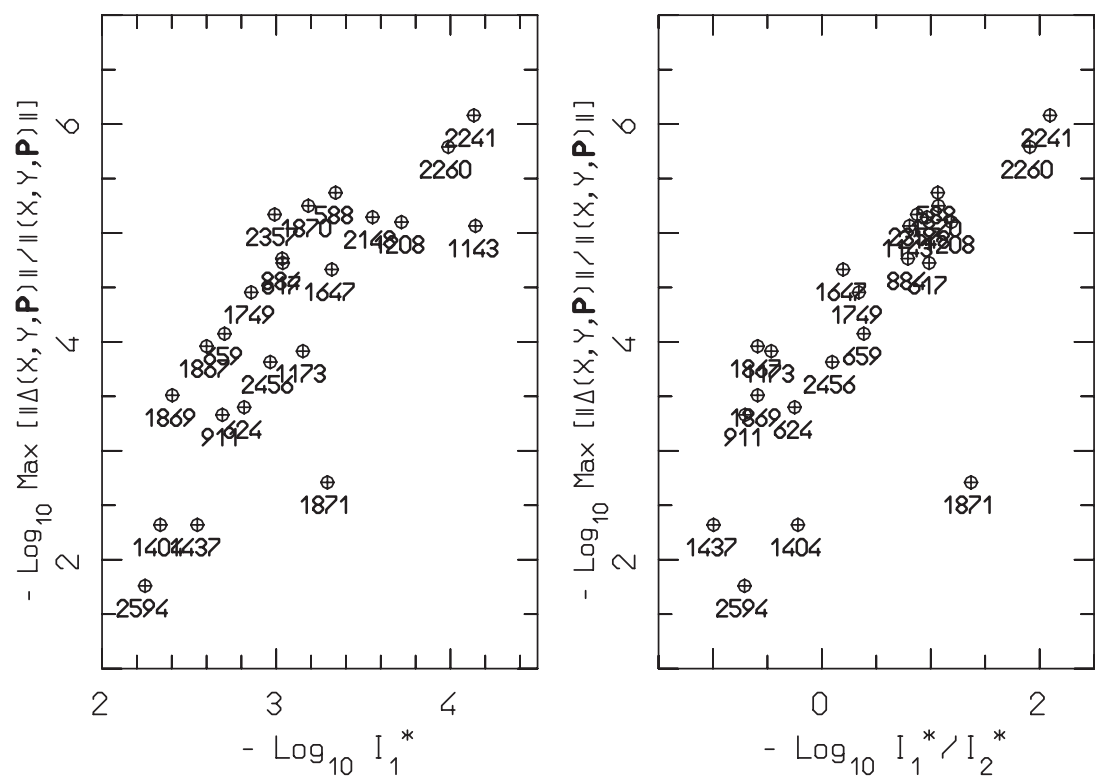

Figure 11. The maximum of the relative difference on $\|(X, Y, \mathbf{P})\|$ as a function of the first component of the initial translation (on the left) and of the ratio of the two components (on the right) for all the asteroids for which our algorithm ends successfully.

essentially ruled by the translation vector, as discussed in point (ii) of section 4.1. Comparing the third column with the fourth and the fifth ones, one can appreciate that the bigger $\bar{I}$ is, the slower the convergence of the algorithm. Note that the distance in the direction $I_{1}$ has a larger weight compared with $I_{2}$.

In the last column we report the maximum for $t \in\left[-10^{5}, 10^{5}\right]$ of the relative difference $\Delta(X(t), Y(t), \mathbf{P}(t)) /\|(X(t), Y(t), \mathbf{P}(t))\|$ between the values of $(X, Y, \mathbf{P})$ calculated by the numerical integration of the RTBP and those given by the semi-analytic one, based on the scheme (30). Let us remark that the relative difference is always nearly aligned in the direction of the second component (in terms of the polar coordinates, this corresponds to the angular variable). The agreement between the two methods is not always as good as in the case of 588 Achilles, represented in figure 9. Indeed, (the second component of) the maximum of the relative difference ranges between $8 \times 10^{-7}$ and $2 \times 10^{-2}$.

The behaviour of $\operatorname{Max}_{t \in\left[-10^{5}, 10^{5}\right]}[\|\Delta(X(t), Y(t), \mathbf{P}(t))\| /\|(X(t), Y(t), \mathbf{P}(t))\|]$ as a function of either $\bar{I}_{1}$ or $\bar{I}_{1} / \bar{I}_{2}$ is rather regular, as shown in figure 11 . The data corresponding to the asteroids reported in table 1 are mainly located close to the diagonal of the two pictures and, sometimes, they are quite well grouped together (especially in the right plot). The agreement between the two methods is obviously good when the translation vector is small, but, rather surprisingly, the cases with low perturbation (or, equivalently, with a fast convergence to zero of the generating functions) are not those corresponding to the best results: we can see this, for instance, by comparing the rows related to 1173 Anchises and 1647 Menelaus with 2241 Alcathous. In general, when $\bar{I}_{2} \gg \bar{I}_{1}$ (i.e. in the right-hand sides part of the pictures in figure 11), we have an excellent agreement between the two methods. Indeed, there is only one such cases (i.e. 1871 Astyanax, which looks very far from the diagonal in figure 11) where the maximum of the relative difference is larger than $2 \times 10^{-5}$; but the initial conditions of that asteroid are quite far from the equilibrium point, and in that case we remarked that, by calculating slightly smaller expansions of the functions, the final results are clearly 
worse; therefore, we expect that the agreement can be improved by increasing the size of the expansions. Note that the size of the expansions is not only determined by the final number of performed steps of the algorithm. Indeed, there are many parameters ruling the truncations of Hamiltonians and canonical transformations, that will be described in the next section.

The agreement between the semi-analytic integration and the numerical one progressively deteriorates when the value of either $\bar{I}_{1}$ or $\bar{I}_{1} / \bar{I}_{2}$ increases, up to the worst case of 2594 Acamas. Moreover, we have seen that using slightly smaller expansions of the functions to calculate the parametric equations of the final invariant tori, the difference between the two methods of integration remains approximately the same for all the asteroids except for the above discussed case of 1871 Astyanax (in most of the cases the agreement is even better with smaller expansions). These facts lead us to conclude that the main limitation on the precision of the semi-analytic integration should be due to the accumulation of the numerical errors in the calculation of the truncated series approximating the canonical transformations. The size of this error seems to be mainly ruled by the value of $\bar{I}_{1}$.

\section{Details of the computer-assisted proof}

The results described in the previous section greatly improve on the ones in [GS97] and [SD00]. Although our methods and theirs are based on different theorems (KAM and Nekhoroshev, respectively), both sets of results approximate the dynamics of the asteroids using suitable series expansions. Note that none of these results are rigorous mathematical proofs.

The aim of this section is to provide rigorous (computer-assisted) proofs of the convergence of the series produced by our algorithm. As is usual in this kind of computation, the domain of applicability of the results will be significantly smaller. The technique used is taken from [LG00]. For short, we will often refer to the estimates reported there.

The proof is given for a high order expansion of the Hamiltonian. To avoid the control of the estimates for all the canonical transformations of section 4 , we will start the computer-assisted proofs from point (d) of the algorithm described in section 4.3. The last column of table 1 also contains an estimate of the size of the modification introduced by working on a truncated expansion of the RTBP instead of the true RTBP given by (1). Note that for asteroids whose value of $\bar{I}_{1}$ is not too big, the differences due to the change of models are not physically relevant.

We will introduce a further simplification. In order to prove that an orbit cannot diffuse anywhere in the phase space, one should ensure that there is a topological confinement. The basic element for carrying on this 'trapping strategy' is the proof of the existence of families of invariant tori which are close to the initial conditions and have a fixed (Diophantine) ratio of the frequencies $\left(\omega_{1}, \omega_{2}\right)$. Then, the two final trapping tori are given by the intersection of the fixed level of energy with two 'good' families that are selected by trial and error (see [LG00]). In this paper, we will simply prove the existence of some families of invariant tori close to the initial conditions of some asteroids, without completing the trapping method.

Let us now precisely state which is the starting point of our computer-assisted proof. We first did all the canonical transformations described in section 2. Then, we performed the Birkhoff normalization as described in point (i) of section 4.1, by truncating the Hamiltonian up to degree 60 in the actions $I$. Afterwards, we did the initial translation of the actions as at point (ii) of section 4.1 , where the value of the vector $\bar{I}$ for each asteroid is reported in the third column of table 1. In this case, we truncated the Hamiltonian up to degree 20 in the actions $p$ and to degree 42 in the angles $q$. Thus, we derived 23 different initial Hamiltonians (that we indicate with the symbol $\mathcal{H}^{\left(n_{\#}\right)}$ ), and each of them is related to the asteroid with catalogue number $n_{\#}$. For each of them, we tried to prove the existence of a family of tori with frequencies very close to the corresponding ones in the second column of table 1. 
Let us first introduce some notation. If $v \in \mathbb{R}^{n}$, we denote $|v|=\sum_{j=1}^{n}\left|v_{j}\right|$. Also, we write the expansion of a generic function $g \in \mathcal{P}_{l, K}$, with multi-index notation, as $g(p, q)=\sum_{|j|=l} \sum_{|k| \leqslant K}\left[c_{j k} p^{j} \cos (k \cdot q)+d_{j k} p^{j} \sin (k \cdot q)\right]$. Then we introduce the norm

$$
\|g\|=\sum_{|j|=l} \sum_{|k| \leqslant K}\left[\left|c_{j k}\right|+\left|d_{j k}\right|\right] .
$$

We need estimates for the norms of the functions $\mathbf{f}_{l}^{(r, s)}$ and $f_{l}^{(r, s)}$ appearing in

$$
\mathbf{H}^{(r)}(p, q)=\omega \cdot p+\sum_{s \geqslant 0} \sum_{l \geqslant 0} \mathbf{f}_{l}^{(r, s)}(p, q)
$$

and (25), respectively, where the sequences of Hamiltonians $\left\{\mathbf{H}^{(r)}\right\}_{r=1}^{R^{\prime}}$ and $\left\{H^{(r)}\right\}_{r=1}^{R^{\prime \prime}}$ are defined as in points (d) and (e) of section 4.3, starting from $\mathbf{H}^{(1)}=\mathcal{H}^{\left(n_{\#}\right)}$ and from $H^{(1)}=\mathbf{H}^{\left(R^{\prime}\right)}$. More precisely, we select a couple of positive integers $R^{\prime}$ and $R^{\prime \prime}$ (to fix the ideas, in the following we will assume $R^{\prime}=24$ and $R^{\prime \prime}=2048$ ); then, we look for a positive constant $E$ and four finite sequences $\left\{\mathbf{e}_{r}\right\}_{r=1}^{R^{\prime}},\left\{\mathbf{z}_{r}\right\}_{r=1}^{R^{\prime}},\left\{\varepsilon_{r}\right\}_{r=1}^{R^{\prime \prime}}$ and $\left\{\zeta_{r}\right\}_{r=1}^{R^{\prime \prime}}$ of positive real numbers such that

$$
\begin{array}{ll}
\left\|\mathbf{f}_{l}^{(r, s)}\right\| \leqslant \mathbf{e}_{r}^{s} E \mathbf{z}_{r}^{l} \quad \text { for } 1 \leqslant r \leqslant R^{\prime}, \quad s \geqslant 0, \quad l \geqslant 0, \\
\left\|f_{l}^{(r, s)}\right\| \leqslant \varepsilon_{r}^{s} E \zeta_{r}^{l} \quad \text { for } 1 \leqslant r \leqslant R^{\prime \prime}, \quad s \geqslant 0, \quad l \geqslant 0 .
\end{array}
$$

The estimates are obtained as follows:

(a) Estimate of the generating functions $\mathbf{X}_{1}^{(2)}, \mathbf{X}_{2}^{(2)}, \ldots, \mathbf{X}_{1}^{\left(R^{\prime}\right)}, \mathbf{X}_{2}^{\left(R^{\prime}\right)}, \chi_{1}^{(2)}, \chi_{2}^{(2)}, \ldots$, $\chi_{1}^{\left(R^{\prime \prime}\right)}, \chi_{2}^{\left(R^{\prime \prime}\right)}$, and of all the intermediate functions necessary for evaluating the previous ones. This is obtained through a preliminary explicit calculation (by following the prescriptions given in section 4, but using intervalar arithmetic, see, e.g. [KSW96] and [SWW00]) of all the coefficients appearing in the expansions of the functions $\mathbf{f}_{l}^{(r, s)}$ and $f_{l}^{(r, s)}$ with $0 \leqslant l \leqslant 20,1 \leqslant r \leqslant R^{\prime}$ and $0 \leqslant s \leqslant R^{\prime}+1$. Then, we used a scheme of iterative estimates analogous to that described in section 4.1.1 of [LG00]. We just introduced a few obvious changes because here we have to estimate also the terms $\mathbf{f}_{l}^{(r, s)}$ of the Hamiltonians $\mathbf{H}^{(r)}$ generated by the preliminary Kolmogorov normalization algorithm without translations. Following such a procedure, we obtain explicit upper bounds for the functions $\mathbf{f}_{l}^{\left(r^{\prime}, s\right)}, \mathbf{X}_{1}^{(2)}, \mathbf{X}_{2}^{(2)}, \ldots, \mathbf{X}_{1}^{\left(R^{\prime}\right)}, \mathbf{X}_{2}^{\left(R^{\prime}\right)}$ and $f_{l}^{\left(r^{\prime \prime}, s\right)}, \chi_{1}^{(2)}, \chi_{2}^{(2)}, \ldots, \chi_{1}^{\left(R^{\prime \prime}\right)}, \chi_{2}^{\left(R^{\prime \prime}\right)}$, with $0 \leqslant l \leqslant 20,1 \leqslant r^{\prime} \leqslant R^{\prime}, 1 \leqslant r^{\prime \prime} \leqslant R^{\prime \prime}$ and $0 \leqslant s \leqslant R^{\prime \prime}+1$.

(b) Derivation of the upper bounds (33) on the infinite sequence of terms appearing in the expansions (32) and (25). Here, we have found it convenient to start by defining

$$
\mathbf{e}_{1}=\max _{j=1,2}\left\{\left\|\frac{\partial \mathbf{X}^{(2)}}{\partial q_{j}}\right\|\right\}, \quad \mathbf{z}_{1}=\max _{j=1,2}\left\{\frac{1}{\bar{I}_{j}}\right\} .
$$

Then, we select $E$ as the minimum value such that the first inequality in (33) is satisfied for $r=1$ (let us recall that we only have to check a finite number of inequalities, because the Hamiltonian $\mathbf{H}^{(1)}$ is truncated in such a way that $\mathbf{f}_{l}^{(1, s)}=0$ if $l>20$ or $\left.s>21\right)$. We obtained the values of the terms appearing in the finite sequences $\left\{\varepsilon_{r}\right\}_{r=1}^{R^{\prime \prime}}$ and $\left\{\zeta_{r}\right\}_{r=1}^{R^{\prime \prime}}$ by applying iteratively the formulae (46) and (48) of [LG00], starting from $\varepsilon_{1}=\mathbf{e}_{R^{\prime}}$ and $\zeta_{1}=\mathbf{z}_{R^{\prime}}$, while $\left\{\mathbf{e}_{r}\right\}_{r=1}^{R^{\prime}}$ and $\left\{\mathbf{z}_{r}\right\}_{r=1}^{R^{\prime}}$ are analogously derived, by replacing in (46) and (48) of [LG00] the symbols $\varepsilon, \zeta$ with $\mathbf{e}, \mathbf{z}$ and the quantities $\mathcal{G}_{11}^{(r)}, \mathcal{G}_{12}^{(r)}, \mathcal{G}_{21}^{(r)}$ and $\mathcal{G}_{22}^{(r)}$ with $\max _{j}\left\{\left\|\partial \mathbf{X}_{1}^{(r)} / \partial q_{j}\right\|\right\}, 0, \max _{j}\left\{\left\|\partial \mathbf{X}_{2}^{(r)} / \partial q_{j}\right\|\right\}$ and $\max _{j}\left\{\left\|\partial \mathbf{X}_{2}^{(r)} / \partial p_{j}\right\|\right\}$, respectively.

Let us now discuss the results obtained. For three asteroids (namely, 1143 Odysseus, 1173 Anchises and 1647 Menelaus) we have found that the functions generated by the algorithm satisfy the estimates in (33) up to $R^{\prime \prime}=2048$ with the final values of $\varepsilon_{2048}, E$ and $\zeta_{2048}$ reported in table 2 , when the frequencies $\omega_{1}$ and $\omega_{2}$ are subject to the restrictions described in both the second and third columns of table 2. For these three asteroids, we can apply theorem 2 
Table 2. Summary of the upper bounds obtained through a computer-assisted proof. The ratio $-\omega_{1} / \omega_{2}$ is given by its expansion in continued fractions.

\begin{tabular}{|c|c|c|c|c|c|c|}
\hline $\begin{array}{l}\text { Code } \\
\text { number }\end{array}$ & $-\omega_{1} / \omega_{2}$ & Range of $\omega_{1}$ & & $\varepsilon_{2048}$ & $E$ & $\zeta_{2048}$ \\
\hline 1143 & $\begin{array}{l}{[0,12,2,1,1,3,} \\
12,1,4,64,2, \overline{1}]\end{array}$ & $-10^{-10} \leqslant 1+$ & $\frac{\omega_{1}}{0.080453760256} \leqslant 10^{-10}$ & 0.64 & $8.7 \times 10^{-4}$ & $1.7 \times 10^{5}$ \\
\hline 1173 & $\begin{array}{l}{[0,12,1,1,35,2,} \\
2,1,2,133,11, \overline{1}]\end{array}$ & $-10^{-10} \leqslant 1+$ & $\frac{\omega_{1}}{0.079687873098} \leqslant 10^{-10}$ & 0.77 & $3.0 \times 10^{-4}$ & $5.1 \times 10^{4}$ \\
\hline 1647 & $\begin{array}{l}{[0,12,2,4,1,43} \\
\quad 5,2, \overline{1}]\end{array}$ & $-10^{-10} \leqslant 1+$ & $\frac{\omega_{1}}{0.080027652157} \leqslant 10^{-10}$ & 0.75 & $1.8 \times 10^{-3}$ & $2.6 \times 10^{4}$ \\
\hline
\end{tabular}

in [LG00] (proved in [Loc01]) because the threshold value, $\varepsilon^{*}$, calculated as in the statement of that theorem is always grater than $\varepsilon_{2048}$ (i.e. $\varepsilon^{*} \simeq 0.85$ in all three cases). Therefore, we can claim the following.

Theorem. Let us consider the three different conservative dynamical systems related to the Hamiltonians $\mathcal{H}^{\left(n_{\#}\right)}$ (defined at the beginning of this section), where the catalogue number $n_{\#}$ can be equal to 1143, 1173 or 1647. They admit one family of invariant tori each. These three families of tori are such that the fixed ratio of the frequencies $\omega_{1} / \omega_{2}$ and the range of $\omega_{1}$ have the values reported in the second and in the third column of table 2, respectively.

Let us recall that the possibility of applying the KAM-type theorem 2 in [LG00] strongly depends both on the number of steps for which we explicitly compute the functions generated by the algorithm (i.e. $R^{\prime}$ ) and on the number of times we iterate the estimates (i.e. $R^{\prime \prime}$ ), with the help of a computer (see the related discussion in [CGL00]). The choice of these parameters (i.e. $R^{\prime}=24$ and $R^{\prime \prime}=2048$ ) had to take into account the limits imposed by the computing resources available to us, but it has been suitable enough for our purposes.

When we compare the results in table 2 with figure 10, it is clear that the computer-assisted proof has been successful for the asteroids closest to the equilibrium point.

What about the remaining asteroids? We think that a few technical modifications of the iterative estimates should be enough to prove the existence of invariant tori close to the orbits of some asteroids that are near the equilibrium point (namely, 624 Hektor, 1208 Troilus, 2148 Epeios and 2456 Palamedes). The idea is the following: in the cases quoted above, the method fails because, from the iterative formulae (46) and (48) of [LG00], it follows that $\varepsilon_{r} \geqslant \exp \left(\sum_{j=2}^{r} 2 / j^{3}\right) \varepsilon_{1}$, even when the generating functions are very small. Such a problem can be overcome by just skipping a few steps of the standard Kolmogorov normalization (i.e. the one including the translations) and by restarting the algorithm from $r=\bar{r}$ such that

$$
\varepsilon_{1} \simeq\left[\bar{r}^{2}\left(\mathcal{G}_{11}^{(\bar{r})}+\mathcal{G}_{12}^{(\bar{r})}\right) \zeta_{\bar{r}-1}\right]^{1 / \bar{r}}
$$

and

$$
\varepsilon_{1} \simeq\left[\bar{r}^{2} \max \left\{\mathcal{G}_{21}^{(\bar{r})}, 2 \bar{r} \mathcal{G}_{22}^{(\bar{r})}\right\}\right]^{1 / \bar{r}}
$$

On the other hand, we remark that even the sequence of upper bounds generated by the iteration of the estimates in section 4.1.1 of [LG00] (which is previous to the calculation of the sequences $\left\{\varepsilon_{r}\right\}_{r=1}^{R^{\prime \prime}}$ and $\left\{\zeta_{r}\right\}_{r=1}^{R^{\prime \prime}}$, that introduces a further worsening effect) looks clearly divergent for the following asteroids: 2260 Neoptolemus, 1870 Glaukos, 884 Priamus, 659 Nestor, 1404 Ajax and 1869 Philoctetes. A rigorous proof of the existence of tori close to the orbits of these objects, or those located even farther from the equilibrium point, looks 
beyond the limits of our scheme of computer-assisted proof. There are four other asteroids (namely, 588 Achilles, 911 Agamemnon, 1437 Diomedes and 1867 Deiphobus) for which our approach might be successful, but this should require some technical improvements other than those suggested here.

Let us recall that we have not rigorously proved the stability of the motion of any asteroid, because we did not try to ensure the confinement of the orbit by completing the "trapping strategy'. Since the effects of the gravitational attraction exerted by Saturn (and by the other major planets) do strongly change in time the values of the vector $\bar{I}$ related to the various asteroids, we consider that our previous analysis is more interesting than having produced a complete result of stability related to some particular initial conditions. In fact, we think that our discussion shows clearly enough the capabilities and the limits of these techniques.

\section{Conclusions}

In this paper, we have studied the problem of the stability of the Trojan asteroids in the context of the planar RTBP. The basic techniques that we have used are:

(i) The frequency analysis method: to compute the fundamental frequencies for a set of Trojans and to investigate the global dynamics in a neighbourhood of the Lagrangian points.

(ii) A variation of the Kolmogorov normalization algorithm: to construct an invariant KAM torus for each pair of frequencies.

The frequency analysis method helped us to highlight some interesting features of the planar RTBP related to our problem. Indeed, the numerical study near the equilateral points in the action-angle variables $(I, \varphi)$ defined in section 2.2 reveals that the extension of the stability region with respect to the actions, $I$, can become much larger if the angles, $\varphi$, have some particular values. This fact is very unpleasant if one is interested in a perturbative approach, because the coordinates $(I, \varphi)$ are the usual starting point for applying Birkhoff or KAM theory and the size of the perturbation is (roughly) proportional to the norm of the actions. Moreover, the frequency analysis has revealed a further difficulty for such theoretical approaches, i.e. the existence of a manifold (not far from the equilibrium point) where the correspondence between actions and frequencies is degenerate.

The variation in the Kolmogorov normalization algorithm is needed due to the fact that we want to construct invariant tori at some distance from the equilibrium point. The translations of the actions involved in the algorithm are the main source of numerical instability and may prevent the procedure from being convergent. To overcome this inconvenience, first we have constructed an intermediate object (a 'quasi-invariant' torus) without using the translations of the actions and, then, this quasi-invariant torus is used as the starting point of a new Kolmogorov normalization, targeting now the final torus. To implement this strategy in an efficient way, we use a procedure that selects an intermediate torus that is close to the final one. We think that the main source of improvement in the analytic part of this work is due to this new strategy. In fact, our algorithm succeeded in 23 of the 30 cases considered. This corresponds to improving the area of success by a factor close to 100 compared with previous methods (see table 1 of both [GS97] and [SD00]). To further improve our results, we think that a different choice of the initial coordinates could be helpful (see, for instance, [Gar77]). This approach might be investigated in a future work.

We have also seen that our reformulation of the Kolmogorov normalization algorithm can be translated into a computer-assisted proof of existence of invariant tori. We successfully used this technique for the three asteroids closest to the equilibrium point. 
Finally, let us remark that even though we have dealt with an application to a classical problem of celestial mechanics, systems close to an elliptic equilibrium point actually occur in many problems of physics and chemistry. Since our new perturbative approach tries to overcome some difficulties that are peculiar to neighbourhoods of elliptic points and the strategy used here does not exploit in a particular way the features of our actual problem, we believe that our procedure can have interesting applications in many different fields.

\section{Acknowledgments}

The authors thank A Giorgilli and J M Mondelo for letting them use, respectively, a software package for computer-algebra and a program for frequency analysis. P Robutel is also acknowledged for his comments on a previous version of this manuscript. FG and AJ have been supported by the MCyT/FEDER Grant BFM2003-07521-C02-01, the CIRIT grant 2001SGR-70 and DURSI. FG acknowledges the support of the Fulbright-GenCat postdoctoral programme. FG and UL have been supported by the research programme 'Sistemi dinamici non lineari e applicazioni fisiche', prot. no 2001018375_001, financed by MIUR. UL has been supported by the financing programme for young researchers of MURST (no 1707/98) and by the INdAM 2002/03 project 'Sistemi dinamici interagenti'.

\section{References}

[AKN88] Arnold V I, Kozlov V V and Neishtadt A I 1988 Dynamical Systems III, Encyclopaedia of Mathematical Sciences vol 3 (Berlin: Springer)

[Arn63] Arnold V I 1963 Proof of A N Kolmogorov's theorem on the preservation of quasi-periodic motions under small perturbations of the Hamiltonian Russ. Math. Surveys 18 9-36

[Arn64] Arnold V I 1964 Instability of dynamical systems with several degrees of freedom Sov. Math. Dokl. 5 581-5

[Bow] Bowell E The asteroid orbital elements database. Project funded principally by NASA grant NAG54741, and in part by the Lowell Observatory endowment. For more information visit the URL http://www.naic.edu/ nolan/astorb.html

[CG91] Celletti A and Giorgilli A 1991 On the stability of the Lagrangian points in the spatial restricted three body problem Celest. Mech. Dyn. Astron. $5031-58$

[CGL00] Celletti A, Giorgilli A and Locatelli U 2000 Improved estimates on the existence of invariant tori for Hamiltonian systems Nonlinearity $13397-412$

[DDB67] Deprit A and Deprit-Bartolome A 1967 Stability of the triangular Lagrangian points Astron. J. 75 173-9

[DHR67] Deprit A, Henrard J and Rom A R M 1967 Trojan orbits II. Birkhoff's normalization Icarus $6381-406$

[dlL01] de la Llave R 2001 A tutorial on KAM theory Smooth Ergodic Theory and its Applications (Seattle, WA, 1999) (Proc. Symp. Pure Math. vol 69) (Providence, RI: American Mathematical Society) pp 175-292

[dlLGJV05] de la Llave R, González A, Jorba À and Villanueva J 2005 KAM theory without action-angle variables Nonlinearity 18 855-95

[Gar77] Garfinkel B 1977 Theory of the Trojan asteroids I Astron. J. 82 368-79

[GDF $\left.{ }^{+} 89\right]$ Giorgilli A, Delshams A, Fontich E, Galgani L and Simó C 1989 Effective stability for a Hamiltonian system near an elliptic equilibrium point, with an application to the restricted three body problem J. Diff. Eqns 77 167-98

[Gio95] Giorgilli A 1995 Quantitative methods in classical perturbation theory From Newton to Chaos: Modern Techniques for Understanding and Coping with Chaos in N-Body Dynamical Systems ed A E Roy and B D Steves (New York: Plenum)

[GJ01] Gabern F and Jorba À 2001 A restricted four-body model for the dynamics near the Lagrangian points of the Sun-Jupiter system Discrete Contin. Dyn. Syst. Ser. B $1143-82$

[GJ05] Gabern F and Jorba À 2005 Effective computation of the dynamics around a two-dimensional torus of a Hamiltonian system $J$. Nonlinear Sci. at press

[GJR04] Gabern F, Jorba À and Robutel P 2004 On the accuracy of restricted three-body models for the Trojan motion Discrete Contin. Dyn. Syst. 11 843-54

[GL97a] Giorgilli A and Locatelli U 1997 Kolmogorov theorem and classical perturbation theory J. Appl. Math. Phys. (ZAMP) 48 220-61 
[GL97b] Giorgilli A and Locatelli U 1997 On classical series expansion for quasi-periodic motions Math. Phys. Electron. J. 3 1-25

[GMS02] Gómez G, Mondelo J M and Simó S 2002 Refined Fourier analysis: procedures, error estimates and applications Preprint 32, University of Barcelona (http://www.maia.ub.es/dsg/2001/)

[Grö60] Gröbner W 1960 Lie-Reihen und Ihre Anwendungen (Berlin: Springer)

[GS97] Giorgilli A and Skokos C 1997 On the stability of the Trojan asteroids Astron. Astrophys. 317 254-61

[Kol54] Kolmogorov A N 1954 On the persistence of conditionally periodic motions under a small change of the Hamilton function Dokl. Acad. Nauk. SSSR 98 527-30

[KSW96] Koch H, Schenkel A and Wittwer P 1996 Computer-assisted proofs in analysis and programming in logic: a case study SIAM Rev. 38 565-604

[Las95] Laskar J 1995 Frequency map analysis of an Hamiltonian system Workshop on Nonlinear Dynamics in Particle Accelarators (Arcidosso, Italy, 1994), AIP Conf. Proc. 344 130-59

[Las99] Laskar J 1999 Introduction to frequency map analysis Hamiltonian Systems with Three or More Degrees of Freedom (NATO ASI) ed C Simó (Dordrecht: Kluwer) pp 134-50

[Leo62] Leontovich A M 1962 On the stability of the Lagrange periodic solutions of the restricted problem of three bodies Sov. Math. Dokl. 3 425-8

[LG00] Locatelli U and Giorgilli A 2000 Invariant tori in the secular motions of the three-body planetary systems Celest. Mech. Dyn. Astron. 78 47-74

[Loc01] Locatelli U 2001 Proof of a KAM theorem on the existence of invariant tori close to an equilibrium point Quaderni del Dipartimento di Matematica 5, University of Milan

[LR01] Laskar J and Robutel P 2001 High order symplectic integrators for perturbed Hamiltonian systems Celest. Mech. Dyn. Astron. 80 39-62

[Mar71] Markeev A P 1971 Stability of the triangular Lagrangian solutions of the restricted three-body problem in the three-dimensional circular case Sov. Astron. AJ 15 682-6

[Mar73] Markeev A P 1973 On the stability problem for the Lagrange solutions of the restricted three-body problem. J. Appl. Math. Mech. 37 713-17

[MG95] Morbidelli A and Giorgilli A 1995 Superexponential stability of KAM tori J. Stat. Phys. 78 1607-17

[MH92] Meyer K R and Hall G R 1992 Introduction to Hamiltonian Dynamical Systems and the N-Body Problem (New York: Springer)

[Mos62] Moser J 1962 On invariant curves of area-preserving mappings of an annulus Nachr. Akad. Wiss. Goett. H Math.-Phys. Kl. 2 1-20

[Mos68] Moser J 1968 Lectures on Hamiltonian systems Mem. Am. Math. Soc. 81 1-60

[MS81] McKenzie R and Szebehely V 1981 Nonlinear stability around the triangular libration points Celest. Mech. Dyn. Astron. 23 223-9

[MS86] Meyer K R and Schmidt D S 1986 The stability of the Lagrange triangular point and a theorem of Arnold J. Diff. Eqns 62 222-36

[Nek77] Nekhoroshev N N 1977 An exponential estimate of the time of stability of nearly-integrable Hamiltonian systems Russ. Math. Surveys 32 1-65

[PM03] Pérez-Marco R 2003 Convergence or generic divergence of the Birkhoff normal form Ann. Math. 157 $557-74$

[Pös82] Pöschel J 1982 Integrability of Hamiltonian systems on Cantor sets Commun. Pure Appl. Math. 35 653-95

[RGJ05a] Robutel P, Gabern F and Jorba A 2005 The observed Trojans and the global dynamics around the Lagrangian points of the Sun-Jupiter system Celest. Mech. Dyn. Astron. at press

[RGJ05b] Robutel P, Gabern F and Jorba A 2005 The resonant structure of Jupiter's Trojan asteroids and its evolution, in preparation

[RL01] Robutel P and Laskar J 2001 Frequency map and global dynamics in the Solar System I Icarus 152 4-28

[SD00] Skokos C and Dokoumetzidis A 2000 Effective stability of the Trojan asteroids Astron. Astrophys. 367 729-36

[Sev03] Sevryuk M B 2003 The classical KAM theory at the dawn of the twenty-first century Moscow Math. J. 3 1113-44

[Sim89] Simó C 1989 Estabilitat de sistemes Hamiltonians Mem. Real Acad. Cienc. Artes Barcelona $48303-48$

[SWW00] Schenkel A, Wehr J and Wittwer P 2000 Computer-assisted proofs for fixed point problems in Sobolev spaces Math. Phys. Electron. J. 6 1-67

[Sze67] Szebehely V 1967 Theory of Orbits (New York: Academic) 